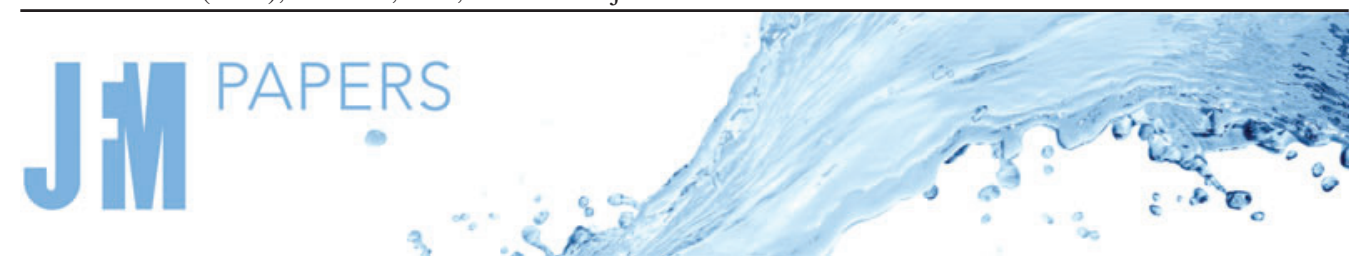

\title{
Impact of a forward-facing step on the development of crossflow instability
}

\author{
Alberto F. Rius-Vidales ${ }^{1} \dagger$ and M. Kotsonis ${ }^{1}$ \\ ${ }^{1}$ AWEP Department, Section Aerodynamics, Delft University of Technology, Kluyverweg 1, \\ 2629HS Delft, The Netherlands
}

(Received 3 December 2020; revised 7 May 2021; accepted 28 May 2021)

The impact of a forward-facing step (FFS) on the development of stationary crossflow instability is investigated on a swept wing model in a low-turbulence wind tunnel at chord Reynolds number of $2.3 \times 10^{6}$. Infrared thermography and particle image velocimetry measurements are used to quantify the transition location and growth of the crossflow instability under the influence of FFSs with different heights. Forced monochromatic stationary crossflow vortices experience an abrupt change in their trajectory as they interact with the step geometry. As the boundary layer intercepts the step an increase in the vertical velocity component and an amplification of the crossflow vortices is observed. Near the step, the vortices reach maximum amplification, while dampening downstream. The smaller FFS cases, show a local stabilising effect on the primary stationary mode and its harmonics, while in the higher step cases transition occurs. The analysis of the temporal velocity fluctuations shows a reduction in the region associated with the type-III travelling crossflow modes downstream of the step. In contrast, the velocity fluctuations in the region associated with type-I secondary instabilities increase past the FFS edge. Nonetheless, in the shortest FFS cases, these velocity fluctuations eventually decay below the clean configuration (i.e. without an FFS) levels. This behaviour is linked to a novel transition delay effect for the shortest step height investigated. The findings highlight new physical aspects driving the interaction between an amplified stationary crossflow vortex and an FFS and provide insight into possible transition delay mechanisms using such geometries.

Key words: boundary layer stability, transition to turbulence, boundary layer control

$\dagger$ Email address for correspondence: a.f.riusvidales@tudelft.nl

(C) The Author(s), 2021. Published by Cambridge University Press. This is an Open Access article, distributed under the terms of the Creative Commons Attribution licence (http://creativecommons.org/ licenses/by/4.0/), which permits unrestricted re-use, distribution, and reproduction in any medium, provided the original work is properly cited. 


\section{A.F. Rius-Vidales and M. Kotsonis}

\section{Introduction}

The rising social awareness of the environmental footprint of commercial aviation is continuously driving research towards more energy-efficient high-subsonic transport aircraft. A significant increase in the aircraft aerodynamic performance can be attained by reducing the skin-friction drag component, owing to its contribution to approximately half of the total drag budget (Joslin 1998; Schrauf 2005; Arnal \& Archambaud 2008; Saric, Carpenter \& Reed 2011). Exploiting the fact that at comparable Reynolds numbers a laminar boundary layer will produce significantly less skin-friction drag than a turbulent boundary layer, an attractive solution is to apply laminar flow control (LFC) techniques to stabilise the boundary layer flow and extend the regions of laminar flow on the outer surface of the aircraft. An in-depth historical review of these techniques has been compiled by Joslin (1998), while more recent updates can be found in Arnal \& Archambaud (2008) and Saric et al. (2011).

The idea of LFC pivots on the stabilisation of the boundary layer against the growth of various instabilities such as Tollmien-Schlichting (TS) waves, attachment-line contamination (ALC), Görtler vortices and crossflow (CF) vortices. The unstable growth of any of these instabilities leads to an anticipation of the laminar-turbulent transition. A comprehensive review of available control strategies for each of these instabilities is provided by Saric et al. (2011).

Particularly pertinent to swept wings, widely employed by modern transport aircraft, is the crossflow instability (CFI) which develops as a series of corotating vortices closely aligned with the external inviscid flow direction. The nature of these vortices (i.e. stationary or travelling) is highly influenced by the level of external disturbances outside the boundary layer flow (Downs \& White 2013). A thorough review of the efforts and results in the study of this instability is provided by Bippes (1999) and Saric, Reed \& White (2003). In the last two decades, research efforts resulted in a considerable advance in the understanding and controlling of the CFI. A summary of different LFC techniques for CFI cases can be found in Messing \& Kloker (2010), Serpieri, Yadala \& Kotsonis (2017) and Saric et al. (2019).

Despite the promising technological advancements in LFC, its performance is highly dependent on the smoothness of the aerodynamic surface, which in practical applications is far from ideal. Manufacturing requirements and operational conditions lead to two-dimensional (i.e. panel joints, seals and seams) and three-dimensional (i.e. rivets, debris, insect contamination) surface features which reduce the effectiveness of LFC techniques by perturbing the boundary layer flow and promoting a premature laminar-turbulent boundary layer transition. This is further exacerbated in swept wings by the inherent sensitivity of CFI to surface roughness. Consequently, understanding the impact of surface irregularities on boundary layer transition is of paramount importance for implementing active/passive LFC techniques in the aviation industry.

Two-dimensional (i.e. spanwise invariant) surface irregularities in the form of backward-facing (BFS) and forward-facing (FFS) steps and gaps, have been a long-standing topic of interest for the design of laminar flow components. The need for a universal method to determine the critical (i.e. transition advancement) step height or gap geometry has driven numerous research efforts on cases where TS waves dominate the laminar-turbulent transition. As part of the X-21A demonstration program (Gerhardt 1967), early wind tunnel experiments on a flat plate model with steps and gaps were conducted. The results indicate that the geometrical limits of surface irregularities compatible with laminar flow could be determined through a critical Reynolds number $\left(R e_{k}\right)$ based on the step height or gap width. Subsequent investigations conducted by 


\section{FFS impact on the development of crossflow instability}

Holmes et al. (1985) on a T34-C aircraft extended the $R e_{k}$ criteria to consider different step-edge shapes (i.e. rounded edge or chamfered edge) for FFS and BFS. The results indicate that by modifying the step-edge geometry, an increase in the critical $R e_{k}$ can be obtained. Additional flight tests by Zuniga et al. (1994) and Drake et al. (1996) studied the effect of step-gap configurations on the laminar-turbulent boundary layer transition of an unswept natural laminar flow (known as NLF) leading edge fixture mounted beneath an F104G aircraft. More recently, Drake, Bender \& Westphal (2008) and Drake et al. (2010) systematically studied the pressure gradient effect on the $R e_{k}$ criterion by using different unswept models in a low-speed wind tunnel and a novel towing facility. The results highlight the dependence of the $R e_{k}$ criterion on the pressure gradient and show the stabilising effect of a favourable pressure gradient on the boundary layer flow in cases with steps.

An alternative criterion for the determination of manufacturing tolerances for laminar flow components is the use of $\Delta N$-factor models. These models aim at incorporating the influence of surface irregularities in the widely used $e^{N}$ transition prediction method. Wang \& Gaster (2005) conducted wind tunnel experiments on FFS and BFS on the surface of an unswept flat plate model with zero pressure gradient at low turbulence conditions. The results indicate a correlation between the reduction in the transition $N$-Factor and the relative step height $\left(h / \delta^{*}\right)$. Moreover, a distinct laminar-turbulent boundary layer transition behaviour was observed between FFS and BFS. In these experiments, FFS showed a less detrimental effect on the boundary layer flow than BFS for the same step height and wind tunnel conditions. Furthermore, Costantini, Risius \& Klein $(2015,2018)$ experimentally investigated the effect of pressure gradient, surface temperature and Mach number on the transition behaviour of FFS on an unswept wing model in a cryogenic Ludwieg-tube wind tunnel.

A number of investigations have been conducted at the French Aerospace Laboratory (ONERA) to develop $\Delta N$-factor models based on numerical simulations and experimental validation. The main results regarding step configurations are presented in Perraud \& Seraudie (2000), Arnal \& Archambaud (2008) and Perraud, Arnal \& Kuehn (2014). A comprehensive review and extension of the work dedicated exclusively to gaps is provided by Beguet et al. (2017). The results presented by Perraud et al. (2014) indicate that the influence of an FFS on the stability of the boundary layer flow strongly differs from the one of a BFS of comparable height. Therefore, to account for these differences, the $\Delta N$-factor in FFS cases is not modelled with a constant shift of the stability curve, but instead a more complex $\Delta N$ model is proposed.

In a different strategy, Crouch, Kosorygin \& Ng (2006) experimentally determined $\Delta N$-factor models based on the change in the laminar-turbulent boundary layer transition location induced by steps on the surface of an unswept flat plate model under favourable and adverse pressure gradients. Crouch \& Kosorygin (2020) extended these empirical models to consider variations in the step location, two-dimensional strips (i.e. FFS followed by BFS) and shallow gaps. In addition, Crouch, Kosorygin \& Sutanto (2020) studied in a similar manner the effects of gaps in the laminar-turbulent boundary layer transition on TS-dominated cases.

Recent numerical studies by Edelmann \& Rist (2015) and Zahn \& Rist (2016) have provided important insight into the boundary layer stability modifications induced by two-dimensional surface irregularities for the further development of $\Delta N$-factor models for unswept geometries. Furthermore, numerical simulations by Rizzetta \& Visbal (2014) on an unswept flat plate geometry with FFS and BFS, elucidated important aspects of the step-induced transition mechanisms dominated by TS waves. 


\section{A.F. Rius-Vidales and M. Kotsonis}

The limited applicability of the aforementioned studies to swept wing cases dominated by CFI, led to parametric studies (Perraud \& Seraudie 2000; Duncan et al. 2014a; Rius-Vidales \& Kotsonis 2020) and more detailed investigations (Saeed, Mughal \& Morrison 2016; Tufts et al. 2017; Eppink et al. 2018; Cooke et al. 2019; Eppink 2020b) on the interaction of $\mathrm{CF}$ vortices with steps configurations. The results from these studies highlight a complex interaction, which occurs when surface irregularities in the form of steps interact with the CFI. Furthermore, previous studies (Perraud \& Seraudie 2000; Tufts et al. 2017) on CFI indicate that configuring a step as a forward-facing arrangement instead of a backward-facing one will result in a weaker destabilisation of the subsonic boundary layer. Thereby, the present work encompasses the detailed study of the impact of a surface irregularity in the form of an FFS on a CFI dominated scenario. Henceforth, the main efforts in the study of this type of surface irregularity in swept geometries are discussed to highlight the unresolved aspects which the present study aims to clarify.

In the last decade, the research group at the Flight Research Laboratory and the Computational Stability and Transition Laboratory of the Texas A\&M university investigated numerically and experimentally (wind tunnel and flight tests) different aspects of swept wing transition under the influence of step surface irregularities. A summary of these efforts and their results is provided in Tufts et al. (2017). Duncan et al. (2014a) conducted experiments on a swept wing boundary layer at low turbulence levels and demonstrated that the interaction between the FFS and the stationary CFI lead to the amplification of the $\mathrm{CF}$ vortices downstream of the step position. More recently Tufts et al. (2017), performed detailed numerical simulations complementary to the flight and wind tunnel experiments presented by Duncan et al. $(2014 a, b)$ and Crawford et al. (2015a). The numerical investigation confirmed the amplification of the incoming stationary CF vortices by the FFS for cases above a critical step height. Tufts et al. (2017) suggested that, due to the spanwise pressure gradients in aft swept wings, the localised recirculation regions upstream and downstream of the step form helical vortices, which travel along the span of the wing. The transition advancement and amplification of the stationary CFI modes were attributed to a constructive interaction between the CF vortices and the downstream helical vortex (i.e. past the step edge) when the FFS height exceeds the core height of the CF vortices. Based on this interaction, Tufts et al. (2017) proposed the use of the unperturbed (i.e. no FFS) CF vortices' core height as a governing metric to determine $a$ priori the criticality of a given FFS.

Although the idea of using the CF vortices' core height as a metric provides a first-order approximation of the critical FFS height (Tufts et al. 2017; Rius-Vidales et al. 2018), further experimental investigations (Eppink \& Casper 2019; Eppink 2018, 2020b), which confirmed the amplification of stationary CFI modes by the FFS, found no evidence to support the constructive interaction proposed by Tufts et al. (2017). Moreover, these experiments revealed that the stationary $\mathrm{CF}$ vortices experience an amplification at two distinct locations in the vicinity of the FFS.

More specifically, Eppink (2020b) studied the mechanisms involved in the FFS-CFI interaction and proposed that the first region of linear growth is caused by the adverse pressure gradient near the step which results in strong inflectional profiles which destabilise the stationary CFI modes. The second region appears to be nonlinearly generated due to the modulation of the recirculation region downstream of the FFS edge, which resulted in streamwise oriented vortices which amplify the harmonics of the primary mode. In addition, the results showed that a subcritical FFS case could lead to a premature boundary layer transition if the initial amplitude of the CF vortices was increased. This effect was attributed to a stronger spanwise modulation of the recirculation region downstream the step edge for the cases with larger initial amplitude. 


\section{FFS impact on the development of crossflow instability}

Furthermore, recent experimental investigations conducted by Rius-Vidales \& Kotsonis (2020) indicate that local one-parameter correlations based on the estimated CF vortex core height or relative step height $\left(h / \delta^{*}\right)$ might not be sufficient to universally capture the influence of an FFS on the laminar-turbulent transition in cases dominated by CFI. A causal relation was identified between the influence of the FFS on the transition process and the stability characteristic of the incoming stationary CFI mode. The results revealed that at fixed ambient conditions (i.e. free stream velocity and turbulence intensity) and initial amplitude of the CF vortices, a given FFS height would result in a subcritical behaviour when forcing an early growth CFI mode (i.e. shorter wavelength than the most amplified at the FFS position) and a supercritical one when forcing a late growth CFI mode (i.e. longer wavelength than the most amplified at the FFS position). In addition, the results show that the addition of the FFS could result in complex nonlinear interactions. In agreement with Eppink \& Casper (2019) and Eppink (2020b) an amplification of the primary mode harmonics was observed downstream of the FFS edge.

Finally, to mitigate the effect of an FFS on transition Eppink \& Casper (2019) successfully applied on a flat plate model dominated by stationary CFI the step-edge chamfering (i.e. slanted step face) strategy by Holmes et al. (1985). Eppink \& Casper (2019) showed that a variation in the angle of the FFS face reduced the recirculation region and $\mathrm{CF}$ reversal. This topological change leads to a weaker destabilisation of the primary CFI mode and a transition postponement with respect to the straight FFS (i.e. vertical step face). In a different strategy, Rizzetta \& Visbal (2017) conducted numerical investigations to manipulate and control the adverse effects of discrete surface irregularities on the CFI development using a plasma actuator. More recently, Ivanov \& Mischenko (2019) used a series of two-dimensional strips (i.e. FFS followed by a BFS) along the span of a swept wing to stabilise the CFI following the theoretical analysis and concept presented by Ustinov \& Ivanov (2018). The experiments by Ivanov \& Mischenko (2019) showed a postponement of the laminar-turbulent transition for strips oriented parallel to the leading edge and at 18 degrees with respect to the leading edge.

The aforementioned studies and the discrepancies between the interaction mechanics proposed by Tufts et al. (2017) and Eppink (2020b) highlight numerous unresolved aspects which require further study before the mechanisms of interaction between the FFS and CF vortices can be fully unveiled. Specifically, the dependence on the initial amplitude of the $\mathrm{CF}$ vortices, nonlinear interactions at the FFS and laminar breakdown mechanisms are key features, requiring experimental and numerical analysis within a range of governing parameters. In addition, except for the recent detailed measurements of Eppink (2020b), experimental identification of velocity and instability development in the vicinity of the step is mostly unavailable, and reconciliation between local to the FFS effects and global transition location is still absent from the published literature.

The objective of this work is to enable a detailed description of the FFS impact on the local development and amplification of the CFI and its effects on the global transition location. Towards establishing a representative interaction scenario, the present study focuses on cases where stationary CF vortices feature a high amplification level upstream of the FFS. The interaction mechanics in these conditions are unveiled by characterising in detail the laminar-turbulent boundary layer transition behaviour and quantifying the local development of the CFI through an exhaustive flow diagnostics study using infrared (IR) thermography and particle image velocimetry (PIV), respectively.

The structure of this paper is as follows. Section 2 provides a detailed description of the wind tunnel facility, swept wing model, the aerodynamic add-ons designed to produce two-dimensional irregularities on its surface, and the measurement technique. Section 3 


\section{A.F. Rius-Vidales and M. Kotsonis}

provides a general overview of the laminar-turbulent transition behaviour and topology induced by the FFS. Section 4 presents an analysis of the impact of an FFS on the development of the CFI.

\section{Experimental set-up and methodology}

This section presents the experimental set-up and methodology by providing a brief overview of the wind tunnel facility, swept wing model and FFS surface irregularities. A description of the measurement techniques and flow metrics is also provided.

\subsection{Wind tunnel facility, swept wing model and surface irregularities}

Experiments have been conducted at the atmospheric closed return low-turbulence tunnel (known as LTT) located at the Delft University of Technology. The wind tunnel features a contraction ratio of $17: 1$. The design and construction of the wind tunnel results in a low turbulence intensity level $\left(T u=\left(1 / U_{\infty}\right) \sqrt{\left(U^{\prime 2}+V^{\prime 2}\right) / 2} \leqslant 0.03 \%\right.$, single hot-wire measurement, bandpass filtered between 2 and $5000 \mathrm{~Hz}$ ) at the nominal conditions employed in this study (Serpieri 2018). All reported measurements were performed on the pressure side of the wing, for a fixed angle of attack of $\alpha=3^{\circ}$ and a Reynolds number of $\operatorname{Re}_{c_{X}}=2.3 \times 10^{6}$, based on the free stream velocity $\left(U_{\infty}\right)$ and the streamwise chord length $\left(c_{X}\right)$. To be noted, the free stream velocity and corresponding Reynolds number used throughout the present work refer to a corrected wind tunnel velocity of $26.5 \mathrm{~m} \mathrm{~s}^{-1}$ based on a calibrated static pressure drop across the tunnel contraction. Due to blockage effects, the free stream velocity just upstream of the wind tunnel model was measured at $27.5 \mathrm{~m} \mathrm{~s}^{-1}$ using a Pitot-static tube.

The M3J swept wing model designed by Serpieri \& Kotsonis (2015), was installed in the wind tunnel octagonal test section $(2.6 \mathrm{~m} \times 1.80 \mathrm{~m} \times 1.25 \mathrm{~m}$; length $\times$ width $\times$ height $)$. Extensive research conducted on the development of CFI (Serpieri \& Kotsonis 2015, 2016), boundary layer control (Serpieri et al. 2017; Serpieri \& Kotsonis 2018; Yadala et al. 2018) and surface irregularities (Rius-Vidales \& Kotsonis 2020) has shown that the combination of this wind tunnel and model offers the necessary conditions for the study of the development of stationary CFI and ensuing transition.

The swept wing model features a streamwise chord of $c_{X}=1.27 \mathrm{~m}$, a span of $b=$ $1.25 \mathrm{~m}$ and a sweep angle of $45^{\circ}$. The airfoil shape, a modified symmetric NACA 66018 (figure $1 a$ ) is designed to enhance the amplification of CFI while suppressing TS waves, Görtler type instabilities and attachment line contamination as shown by Serpieri \& Kotsonis (2016) at mild angles of attack $\left(\alpha \approx 3^{\circ}\right)$. Furthermore, during the experiments, considerable care was taken to ensure a consistent and polished surface near the leading edge, due to the extreme sensitivity of CFI to surface roughness. To this end, a Mitutoyo SJ-310 profilometer was used to examine the swept wing surface. The measured root mean square surface roughness is $R_{q}=0.20 \mu \mathrm{m}$.

The static pressure distribution on the model was measured using a multichannel scanner connected to two rows of 46 streamwise $(X)$ oriented taps at $24 \%$ and $76 \%$ of the model span. At the nominal conditions of this study $\left(\operatorname{Re}_{c_{X}}=2.3 \times 10^{6}, \alpha=3^{\circ}\right)$ the streamwise pressure distribution (pressure side of the wing) shows a favourable gradient up to $X / c_{X} \approx 0.65$ as shown in figure $1(b)$. The nearly invariant pressure along the span, in agreement with Serpieri \& Kotsonis (2016), confirms the adequacy of the infinite swept wing assumption for the boundary layer and stability calculations in the measurement region. 

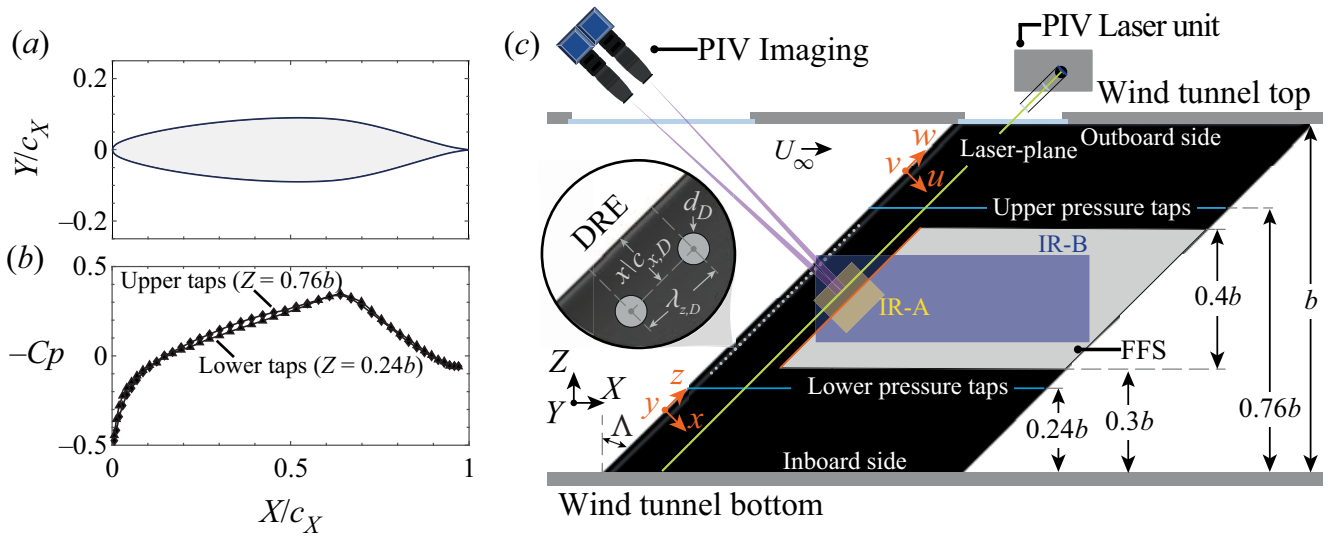

Figure 1. Experimental set-up: (a) swept wing airfoil shape; $(b)$ streamwise pressure coefficient distribution at two spanwise locations measured on the pressure side at $\alpha=3^{\circ}$ and $R e_{c_{X}}=2.3 \times 10^{6}$; (c) general schematic (flow direction from left to right $b=1.25 \mathrm{~m}$ ) showing the FFS location, the IR analysis regions (IR-A, IR-B), planar PIV set-up and details of DRE.

The steady and incompressible 2.5D (i.e. spanwise invariant infinite span conditions) laminar boundary layer equations are solved numerically for the pressure side of the model along the $x$ component (i.e. orthogonal to the leading edge, figure $1 c$ ) using the measured pressure distribution for a reference case with no surface irregularity and no artificial forcing of CFI. The stability of the calculated boundary layer is evaluated by solving the Orr-Sommerfeld equation using the spatial theory formulation, which is simplified by assuming a zero spanwise growth rate $\left(\beta_{i}\right)$ due to the infinite swept wing assumption. Subsequently, the $N$-factor is calculated for a combination of a given spanwise wavelength $\left(\beta_{r}\right)$ and frequency $\omega=0$ (stationary) by integrating the spatial growth rate $\left(\alpha_{i}\right)$ along the $x$-coordinate (Arnal \& Casalis 2000).

As summarised in the previous section, the main objective of the present study is to elucidate the interaction between an FFS irregularity and the incoming stationary $\mathrm{CF}$ vortices. However, the quest for experimentally simulating a representative scenario raises the question regarding which particular mode is more relevant. Several options are available; these entail focusing on the CFI mode most amplified (i.e. highest $N$ ) at the step location (Tufts et al. 2017; Rius-Vidales \& Kotsonis 2020), or the most unstable mode (i.e. highest growth rate) at the step. Another option is to investigate the overall most unstable mode (Tufts et al. 2017; Eppink 2020b). Considering the sensitivity of transition location to step height, the latter option can be further narrowed down to investigating the transition-inducing mode at clean conditions (i.e. no FFS). Following these considerations, the present work is indeed focusing on the transition-inducing mode at clean conditions. Preliminary stability analysis results at the tested conditions, identified a stationary CFI mode featuring a spanwise wavelength close to $\lambda_{z}=7.5 \mathrm{~mm}$ as the most amplified prior to transition and exhibiting a monotonic growth upstream and downstream of the step location $\left(x_{h} / c_{x}=0.2\right)$.

To study the impact of an FFS on this unstable stationary CFI mode, discrete roughness elements (DREs) are used (Reibert et al. 1996; Saric, Carrillo \& Reibert 1998; Serpieri \& Kotsonis 2016) to force a single fundamental CFI mode featuring a nominal spanwise wavelength of $\lambda_{z, D}=7.5 \mathrm{~mm}$ in the vicinity of the leading edge. Motivated by a lack of experimental or numerical evidence pertaining to interaction of strongly amplified CFI with steps and in line with previous experiments (Rius-Vidales \& Kotsonis 2020), DREs 


\section{A.F. Rius-Vidales and M. Kotsonis}

$\begin{array}{lccccc}\text { ID } & \bar{h}(\mu \mathrm{m}) & \sigma_{h}(\mu \mathrm{m}) & x_{h} / c_{x} & \delta_{h}^{*}(\mu \mathrm{m}) & y_{c}(\mu \mathrm{m}) \\ \text { Clean } & - & - & - & 444 & 1029 \\ A_{1-4} & 368 & 3 & 0.150 / 0.175 / 0.200 / 0.225 & - & - \\ B_{3} & 474 & 3 & 0.200 & - & - \\ C_{3} & 579 & 3 & 0.200 & - & - \\ D_{3} & 759 & 8 & 0.200 & - & -\end{array}$

Table 1. Geometrical parameters of tested configurations. For all cases the nominal DRE settings are: $\lambda_{z, D}=7.5 \mathrm{~mm} ; d_{D}=2 \mathrm{~mm} ; k_{D}=100 \mu \mathrm{m} ; x_{D} / c_{x}=0.02$.

with a relatively large nominal height of $k_{D}=100 \mu \mathrm{m}$ and a diameter of $d_{D}=2 \mathrm{~mm}$ were manufactured in-house from an adhesive transfer vinyl film using a custom laser cutting system. The DREs were installed at $x_{D} / c_{x}=0.02$, which is just upstream of the forced mode neutral point according to linear stability theory calculations.

Surface add-ons have been commonly used to create FFS surface irregularities on existing wind tunnel models (Holmes et al. 1985; Perraud \& Seraudie 2000; Rius-Vidales et al. 2018; Rius-Vidales \& Kotsonis 2020). Following a similar approach in this study, a polyethylene terephthalate (known as PET) foil is used to simulate a sharp edge FFS on the surface of the existing swept wing model. Foils of different thicknesses were cut to size using a CNC DCS 2500 Gerber machine, and each was installed on the model, as illustrated by the grey region in figure $1(c)$.

The resulting FFS step height was characterised in situ by traversing a Micro-Epsilon 2950-25 laser profilometer (reference resolution of $2 \mu \mathrm{m}$ ) along $200 \mathrm{~mm}$ of the spanwise extent of the surface irregularity, centred at midspan. Table 1 indicates the resulting average step height $(\bar{h})$, standard deviation $\left(\sigma_{h}\right)$ and streamwise location $\left(x_{h} / c_{x}\right)$ of the FFS surface irregularity for all tested configurations. As an indication of the relative size of the FFS inside the boundary layer flow, the displacement thickness $\left(\delta_{h}^{*}\right)$ is computed based on the aforementioned 2.5D numerical boundary layer solution for the streamwise velocity $(u)$ at the nominal step location $\left(x_{h} / c_{x}=0.2\right)$. Similarly, from the linear stability theory analysis the estimated CF vortex core height $\left(y_{c}\right)$ based on Tufts et al. (2017) at the step location (indicated in table 1) is extracted from the $v$-perturbation eigenfunction corresponding to the CFI mode featuring the spanwise wavelength enforced by the DREs $\left(\lambda_{z, D}=7.5 \mathrm{~mm}\right)$.

Finally, two different coordinate systems are used throughout this study with their origin at the intersection between the leading edge and the wing midspan. As illustrated in figure $1(c)$, the first coordinate system spatial coordinates $(X, Y, Z)$ and velocity components $(U, V, W)$ are referenced according to the $X$-coordinate being parallel to the wind tunnel floor. On the second coordinate system, the spatial coordinates are given by $(x, y, z)$ and the velocity components $(u, v, w)$. In this case, the $x$-coordinate is perpendicular to the leading edge.

\subsection{Measurement techniques}

\subsubsection{Infrared thermography}

The well known Reynolds analogy predicts an increase in wall shear stress typically associated with the laminar breakdown, which leads to an increase in the surface heat transfer. Therefore, to determine the laminar-turbulent boundary layer transition location in this study, an IR thermographic system has been used. Due to its non-intrusiveness, this 


\section{FFS impact on the development of crossflow instability}

measurement technique has been successfully employed for transition identification in the study of swept wing flows as shown by Zuccher \& Saric (2008), Crawford et al. (2015b) and Rius-Vidales \& Kotsonis (2020).

The model was continuously irradiated during the measurements by seven halogen lamps to increase the thermal contrast on the IR images. Six lamps of $400 \mathrm{~W}$ were located at the optical access ports on the upper and lower parts of the test section and one of $1000 \mathrm{~W}$ at mid-test-section height upstream of the model. The overall variation of surface temperature during the measurements was registered to be less than half a degree, thus ensuring a minimal influence on the near-wall fluid properties.

Two Optris PI640 IR cameras $(640$ pixel $\times 480$ pixel, uncooled focal plane array, 7.5-13 $\mu \mathrm{m}$ spectral range, NETID $75 \mathrm{mK}$ ), designated as IR-A and IR-B, image the pressure side of the model through small openings on the vertical wall of the test section. Camera IR-A equipped with a telephoto lens $(f=41.5 \mathrm{~mm})$ images a region near the step. Conversely, camera IR-B equipped with a wide-angle lens $(f=10.5 \mathrm{~mm})$ images a larger portion of the model as illustrated in figure $1(c)$. The analysis region by camera IR-A has a dimension of $145 \times 200 \mathrm{~mm}$ (centred at $X / c_{X}=0.2$ and $Z / b=0$ ) and for camera IR-B $1000 \times 400 \mathrm{~mm}$ (centred at $X / c_{X}=0.37$ and $\left.Z / b=0\right)$.

The processing and extraction of the transition location from the measurements of camera IR-B were performed using an in-house pattern recognition code. The camera acquires 78 images at $3.5 \mathrm{~Hz}$. For each measurement series, a time-averaged temperature map is calculated, and a physical space transformation and distortion correction is applied to it. Subsequently, a differential infrared thermography technique (DIT) (Raffel \& Merz 2014; Raffel et al. 2015) is applied to consecutive temperature fields with increasing Reynolds numbers. This minimises the background noise and increases the signal to noise ratio during the determination of the transition location following previous experiments by Rius-Vidales \& Kotsonis (2020). Finally, the gradient of the binarised DIT image is calculated, and a linear fit of the transition front is performed for the analysis region. The confidence bands of the fit are proportional to the transition front uniformity (i.e. jagged or smooth).

Figure 1(c) shows that camera IR-A captures, in more detail, the thermal footprint of the flow structures in the vicinity of the FFS. By applying a spatial power spectral density (PSD) analysis on the thermal intensity values along the spanwise $(z)$ component of these measurements, the changes in the spatial organisation and direction of the $\mathrm{CF}$ vortices induced by the FFS are examined. The spatial frequency resolution (i.e. smallest resolved wavelength) stemming from the Nyquist limit of the sensor is $0.86 \mathrm{~mm}$.

\subsubsection{Particle image velocimetry}

Quantitative measurements of the flow dynamics and instability interacting with the FFS have been enabled using planar PIV (2C-2DPIV). Measurements on a $z-y$ plane normal to the surface and parallel to the leading edge (figure $1 c$ ) have been conducted at various chordwise locations $\left(0.17 \leqslant x / c_{x} \leqslant 0.25\right)$. These planes were imaged in streamwise increments of $5 \mathrm{~mm}\left(\approx 0.4 \%\right.$ of $\left.c_{X}\right)$ for the majority of stations. Additionally, increments of $1 \mathrm{~mm}$ were used near the FFS. The translation was enabled using an automated traversing system mounted outside of the wind tunnel test section capable of simultaneously move the imaging and laser unit with a positioning accuracy of $\pm 6.2 \mu \mathrm{m}$.

The laser unit comprises a Quantel Evergreen Nd:YAG dual cavity laser (200 mJ). Through a set of spherical and cylindrical expansion optics, the laser beam is shaped into a plane, with a thickness of $\sim 1 \mathrm{~mm}$. The plane propagates along the $z$ direction (i.e. parallel to the leading edge) as shown in figure $1(c)$. 


\section{A.F. Rius-Vidales and M. Kotsonis}

During the measurements, the wind tunnel is homogeneously seeded with water-glycol droplets (average particle size $1 \mu \mathrm{m}$, particle response time $3.54 \mu \mathrm{s}$ and Stokes number of 0.05 ) using a SAFEX fog generator located in a vent downstream of the test section. The seeding recirculates in the closed circuit of the wind tunnel. Images of seeding particles as they cross through the laser plane are recorded by two LaVision Imager sCMOS camera (sCMOS, $2560 \times 2160$ pixels, 16-bit, $6.5 \mu \mathrm{m}$ pixel pitch) positioned outside the test section as shown in figure $1(c)$. Each camera is equipped with a $f=200 \mathrm{~mm}$ objective operated at $f_{\#}=11$ and two $\times 2$ teleconverters. This configuration yields a large optical magnification factor $(M=0.9)$, necessary for the detailed measurement of the boundary layer flow near the FFS location. The interframe time $(\Delta t)$ was set to $15 \mu$ s which resulted in an average particle displacement in the free stream of 12 pixels.

Each measurement consists of 1200 image pairs acquired at $15 \mathrm{~Hz}$ per camera. For each image pair, a multistage cross-correlation was performed using LaVision Davis 10 with a final interrogation window of $12 \times 12$ pixel $^{2}$ and overlap of $75 \%$. Additionally, the results of both cameras are stitched together to capture the development of two full CF vortices in the measurement region. The final analysis at each measurement location has a dimension of $15 \mathrm{~mm} \times 3 \mathrm{~mm}(W \times H)$, a vector spacing of $20 \mu \mathrm{m}$ and a maximum uncertainty in the time-average results inside the boundary layer for the spanwise $U_{\bar{w}}=0.49 \% w_{e}$ and vertical component $U_{\bar{v}}=0.38 \% w_{e}$.

\subsection{Boundary layer flow stability metrics}

The changes in the stability of the CF vortices induced by the FFS are studied by analysing the streamwise evolution of the experimental spanwise steady disturbance profile based on the time-averaged planar PIV velocity measurements described in $\$ 2.2 .2$.

The methodology described by White \& Saric (2005) and Downs \& White (2013) indicates that the experimental steady disturbance profile is equivalent to the spanwise root mean square $\left(\langle\rangle_{z}\right)$ of the time-average perturbation, which for the spanwise component is given by $\langle\hat{w}(y)\rangle_{z}=\left((1 / n) \sum_{j=1}^{n}\left(w\left(y, z_{j}\right)-\bar{w}(y)\right)^{2}\right)^{1 / 2}$. Subsequently, the streamwise change in the steady disturbance can be determined by calculating the mathematical maximum of the profiles along the $y$-coordinate as $A_{M}=\max \left(\langle\hat{w}(y)\rangle_{z}\right)$.

It is important to note that according to perturbation analysis used in stability theory, the velocity perturbation $\left(w^{\prime}\right)$ should be calculated by subtracting a basic state or baseflow $(W)$ to the mean velocity component $(w)$. Hence, in this context, $\hat{w} \neq w^{\prime}$ since the experimentally measured spanwise average $\bar{w}(y)$ corresponds to a mean-flow distorted flow and not to a baseflow. Nevertheless, this approach has been traditionally used as a metric to determine the growth and decay of CFI modes in experimental conditions and is accordingly followed in the present study as well.

\section{Transition behaviour and topology}

This section presents the influence of an FFS on the laminar-turbulent boundary layer transition. The determination of the transition location and the topology of the developing CF vortices is based on the IR measurements acquired and processed as indicated in $\S 2.2 .1$ for the different configurations indicated in table 1 .

\subsection{Global influence of the FFS on transition location}

The laminar-turbulent boundary layer transition location was determined for each configuration based on the camera IR-B surface thermal maps. The time-averaged thermal 


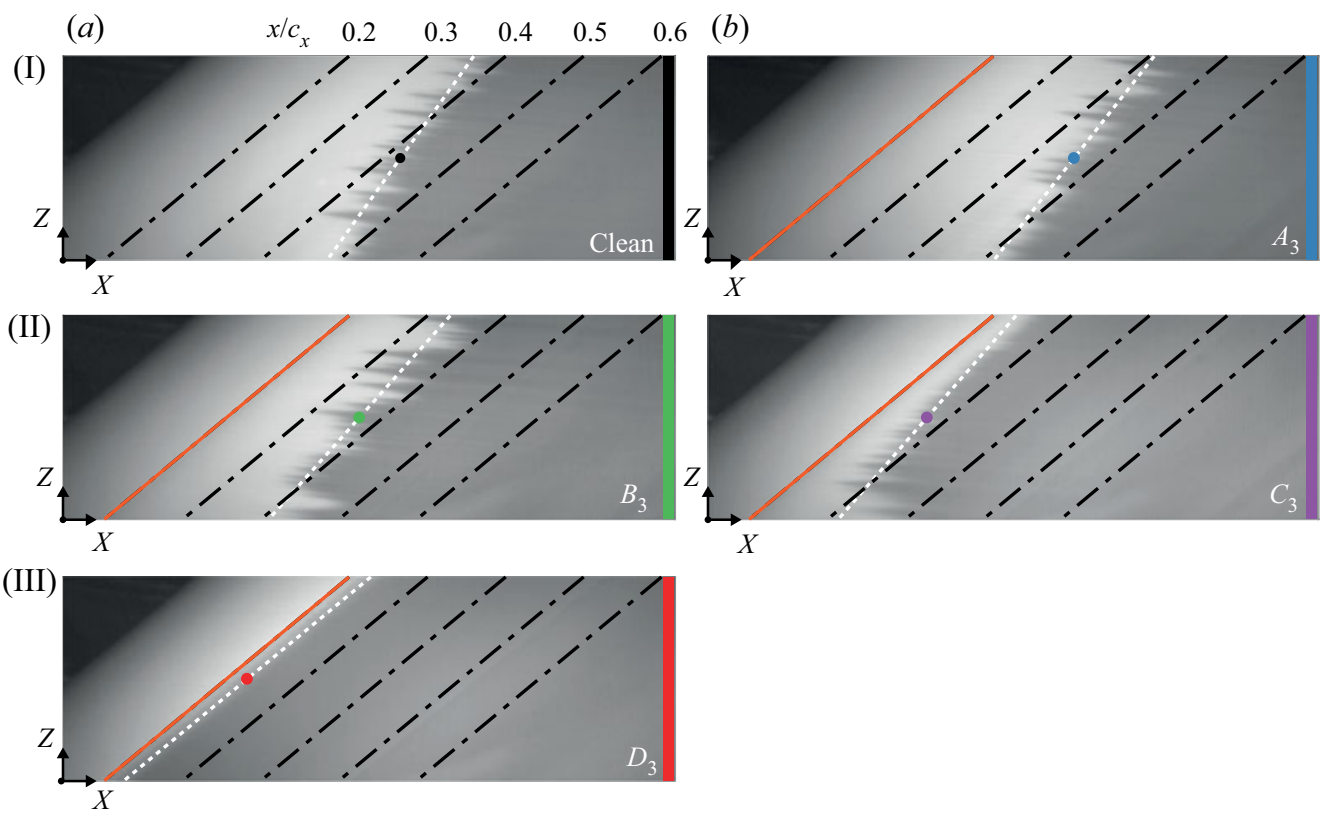

Figure 2. The IR-B thermal maps (flow from left to right) displaying the effect of an FFS (orange line) on the transition front pattern, dashed white line indicates transition linear fit and $(\bullet)$ marks its projection to the mid-domain of the measurement: (I $a$ ) clean; (I $b) A_{3}$; (II $a$ ) $B_{3}$; (II $b$ ) $C_{3}$; (III $\left.a\right) D_{3}$.

maps presented in figure 2(I $a$ ) reveal a jagged transition front pattern which extends along the span of the wing. The nature of this jagged pattern has been traced to the breakdown process typical of stationary CF vortices (Dagenhart et al. 1989; Bippes 1999; Saric et al. 2003; Downs \& White 2013). As the stationary CF vortices saturate, a rapidly growing secondary instability leads to a local breakdown of the CF vortices characterised by contiguous turbulent wedges forming along the span creating the so-called 'jagged' or 'sawtooth' transition front pattern. Therefore, the appearance of this pattern indicates the dominance of stationary CFI modes over travelling modes. In contrast, when the latter dominate, a more smooth (i.e. non-wedged) time-average transition front is observed due to the movement of CF vortices along the span.

The transition front location and variance along the span is identified from the surface thermal maps of camera IR-B following the DIT technique mentioned in $\$ 2.2 .1$ and employed in Rius-Vidales \& Kotsonis (2020). It is particularly noteworthy that the linear fit of the identified transition front locations (white dashed line in figure 2I $a$ ) forms an angle with the leading edge, which reduces with increasing step height. This behaviour is related to the non-uniform wind tunnel blockage along the height of the wind tunnel (i.e. along the $Z$-direction in figure $1 c$ ) which leads to slightly stronger favourable pressure gradients near the outboard side of the wing. Consequently, to quantify the effect of the FFS height the transition location is extracted from the linear fit at the middle of the measurement domain $(\bullet$ marker in figure 2I $a$ ). The results presented in figure 3 show that for the clean configuration (table 1) laminar-turbulent transition occurs at $x_{t} / c_{x} \approx 0.42$ (solid black line in figure $3 a$ ).

The addition of a moderate FFS $\left(B_{3}\right.$ and $C_{3}$ in figure $3 a$ ) results in a critical regime behaviour, as the boundary layer transition front shifts upstream of the clean configuration as illustrated in figure $2(\mathrm{II} a-\mathrm{I} b)$. A further increase in step height $\left(D_{3}\right)$ leads to a 


\section{A.F. Rius-Vidales and M. Kotsonis}

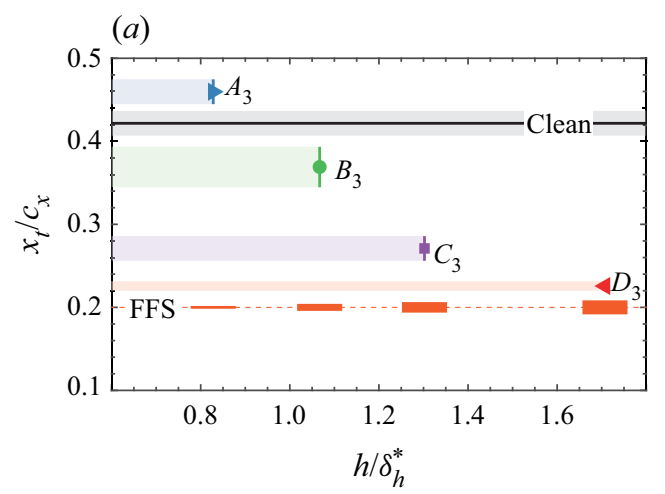

(b)

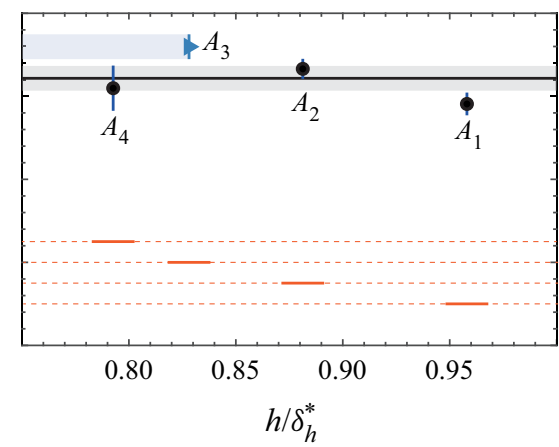

Figure 3. Transition location: ( $a$ ) fixed step $\left(x_{h} / c_{x}=0.2\right)$ location for different FFS; $(b)$ variation of the step location $\left(0.15 \leqslant x_{h} / c_{x} \leqslant 0.225\right)$ for small FFS $(A)$.

supercritical regime behaviour with a substantial reduction in the extent of laminar flow as shown in figure 2(III $a$ ). These results indicate a gradual degradation of the laminar flow with increasing step height analogous to the behaviour observed by Crawford et al. (2015a) and Rius-Vidales \& Kotsonis (2020), and somewhat different to the one presented in Duncan et al. (2014a) and Eppink (2020b) which showed that with increasing step height the transition location more abruptly shifts towards the FFS location.

An important note must be made regarding the use of the terms critical and supercritical, which vary significantly in previous works on roughness effects on transition. Throughout the present study, the term critical is assigned to cases in which the step has an identifiable effect on transition location, while the term supercritical refers to cases in which transition location is very near or at the step location. A supercritical behaviour essentially denotes flow 'tripping' due to the step. Therefore, the critical step height definition used in this work is compatible with the one presented in Tufts et al. (2017).

Notwithstanding the general adverse effects of an increasingly high FFS on transition, it is remarkable to note that a new transition behaviour is revealed when adding a small FFS $\left(A_{3}\right.$ in figure $\left.3 a, b\right)$ at these conditions. Counter-intuitively, the addition of the FFS results in a favourable effect as transition postponement is observed instead of advancement. At first glance, this unexpected result is in disagreement with most of previous experimental or numerical observations on surface irregularities, and common wisdom alike. However, observations recently presented by Ivanov \& Mischenko (2019) based on the concept presented by Ustinov \& Ivanov (2018) suggested a transition delay effect under the influence of rectangular (i.e. FFS followed by a BFS) surface reliefs. Nonetheless, regarding surface irregularities in the form of only FFS the behaviour observed in this work contrast with previous studies (Perraud \& Seraudie 2000; Duncan et al. 2014a; Crawford et al. 2015b; Saeed et al. 2016; Eppink 2017; Tufts et al. 2017; Eppink 2018; Rius-Vidales et al. 2018; Eppink \& Casper 2019; Eppink 2020a,b), as to the best of the authors' knowledge the present case is the first report of a transition delay in a boundary layer dominated by CFI in the presence of an FFS.

Given the novelty of this result and the potential such delay behaviour can offer in a LFC strategy, three additional configurations $\left(A_{1,2,4}\right.$, table 1$)$ have been tested. The objective of such variation was to exclude random and systematic measurement errors that might bias the result, as well as to establish a range of governing parameters for which the transition delay effect is observed. Figure 3(b), shows the effect of varying the streamwise position of this small FFS $(A)$ on the laminar-turbulent boundary layer transition. When the FFS 
(a)
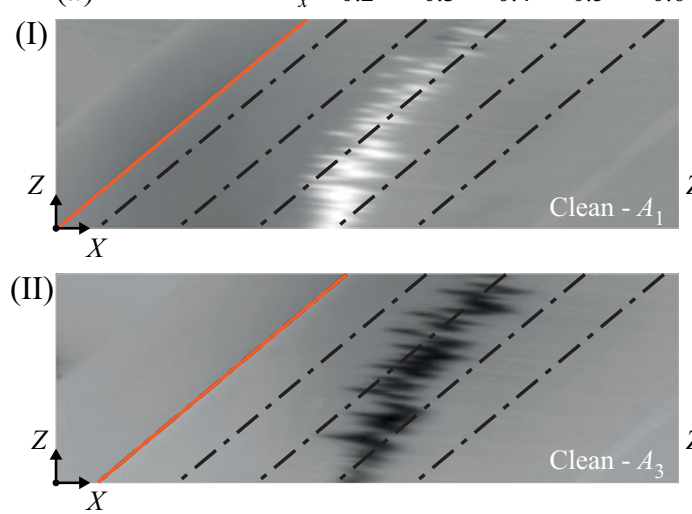

(b)
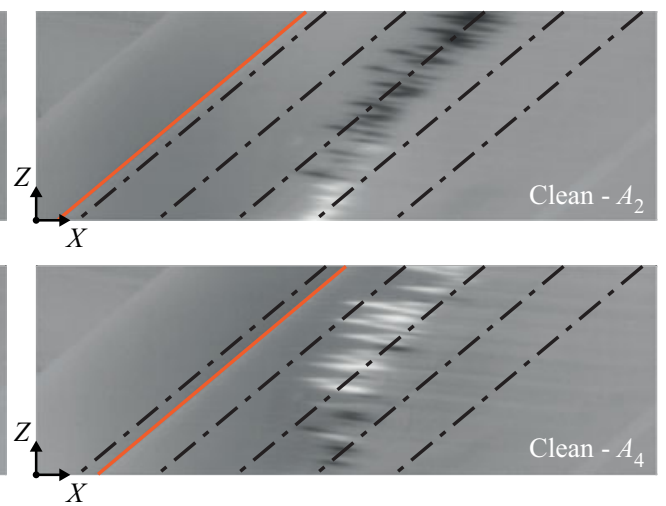

Figure 4. The IR-B differential thermal maps (flow from left to right) displaying transition advance and postponement effects by FFS $A$ (orange line): (I $a) \Delta I_{A_{1}}$; (I b) $\Delta I_{A_{2}}$; (II $\left.a\right) \Delta I_{A_{3}}$; (II $\left.b\right) \Delta I_{A_{4}}$.

is located at the most upstream location $A_{1}$ a slight transition advancement is measured. However, as the FFS is translated downstream the detrimental effect of the step reduces for the case $A_{2}$ until a clear transition delay effect is observed for the position of case $A_{3}\left(x_{t, \text { Clean }} / c_{x}-x_{t, A_{3}} / c_{x}=-0.038\right)$. The consistent behaviour of transition location with the location of the step further confirms the validity and physicality of the transition delay effect. Nevertheless, the non-monotonic trend and rather narrow range of streamwise FFS locations for which the transition delay effect is observed points to the existence of possibly conflicting mechanisms governing the observed transition location.

Towards further probing the effect, a subtraction of thermal maps pertaining to the $A_{1-4}$ FFS positioned at several streamwise locations from the thermal map of the clean case $\left(\Delta I_{A_{1-4}}=I_{\text {Clean }}-I_{A_{1-4}}\right)$ for these conditions are shown in figure 4 . The subtracted thermal maps confirm that the transition delay (dark regions) is not a localised or outlier-dominated effect but occurs over a considerable spanwise extent of the measurement region. Note that the differences in the delay effect on the outboard and inboard section of the wing are again related to the non-uniform blockage along the height of the wind tunnel (i.e. Z-direction figure $1 c$ ).

The global behaviour of transition location with varying FFS height highlights the intricate flow dynamics, which results from the interaction between the FFS and the $\mathrm{CF}$ vortices, necessitating high-resolution velocity measurements for further analysis. Notwithstanding the present observations, caution needs to be exercised when interpreting or generalising these results since, in addition to local parameters (i.e. step height, boundary layer displacement thickness and $\mathrm{CF}$ vortex core height) the influence of the FFS also depends on the stability characteristics of the incoming CFI mode (Rius-Vidales \& Kotsonis 2020).

\subsection{Local influence of the FFS on the organisation of the CF vortices}

The thermal maps of camera IR-A (figure $1 c$ ) provide a more detailed visualisation near the step region by showing the thermal footprint of the CF vortices on the surface of the wing model as alternating streaks of high (i.e. lighter) and low (i.e. darker) temperature as presented in figure $5(\mathrm{I} a)$. This particular temperature distribution originates from a variation in the heat transfer of the near-wall fluid due to a change in the magnitude of local skin friction coefficient induced by the high and low shear regions on the baseflow 


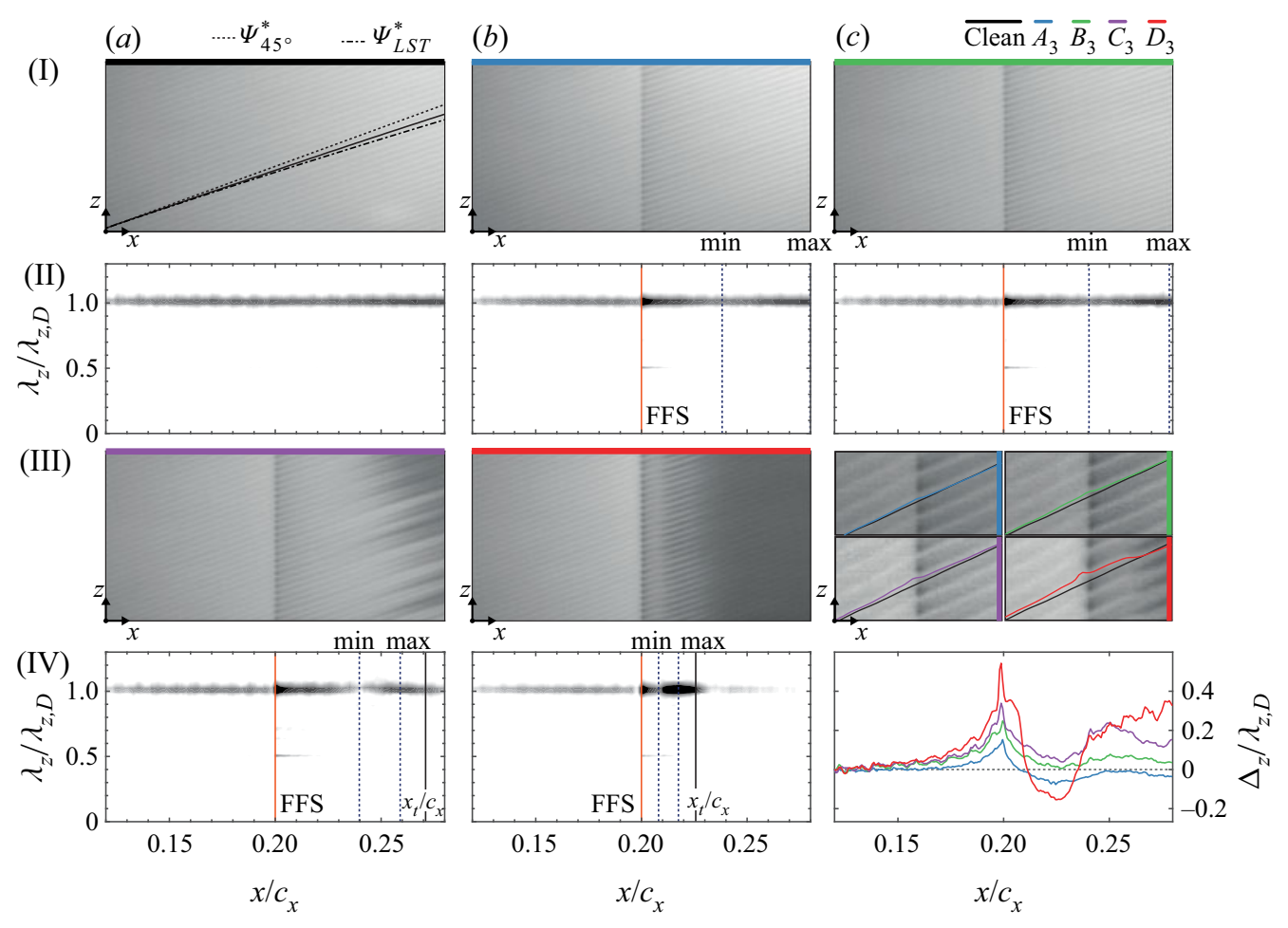

Figure 5. Comparison of IR-A thermal maps (I and III, flow from left to right) and spatial PSD analysis (II and IV, 20 levels of $\ln \left(P / \bar{P}_{\text {max }_{z}}\right)$ from -3 to 1$)$ : (I $a-\mathrm{II} a$ ) clean; (I $\left.b-\mathrm{II} b\right) A_{3}$; (Ic-II $\left.c\right) B_{3}$; (III $\left.a-\mathrm{IV} a\right) C_{3}$; (III $b-\mathrm{IV} b$ ) $D_{3}$; (IIIc) CF vortices trajectories; (IVc) change in CF vortices trajectory due to FFS. (Here $\lambda_{z, D}=7.5 \mathrm{~mm}$ ).

modulated by the CF vortices. Therefore, the local influence of the FFS on the CF vortex thermal footprint is evaluated by calculating the spatial PSD, as indicated in $\S 2.2 .1$, from a series of spanwise (z-direction) temperature profiles extracted at different streamwise locations.

The results for the clean configuration in figure 5(II $a)$, identify a series of CF vortices monotonically spaced at the spanwise wavelength of the forcing DREs $\left(\lambda_{z} / \lambda_{z, D}=1\right.$, $\left.\lambda_{z, D}=7.5 \mathrm{~mm}\right)$ for the entire measurement region. Upon the addition of a small FFS $\left(A_{3}\right.$, figure $\left.5 \mathrm{II} b\right)$ a clear peak at the forced wavelength is observed at the step location $\left(x_{h} / c_{x}=0.2\right.$ and $\left.\lambda_{z} / \lambda_{z, D}=1\right)$. In addition, a second peak centred at the wavelength of the forced mode first harmonic $\left(\lambda_{z} / \lambda_{z, D}=0.5\right)$ at the step location is also present. This behaviour occurs in all step cases in figures $5(\mathrm{II} b-\mathrm{II} c, \mathrm{IV} a-\mathrm{IV} b$ ) and is in agreement with Eppink (2020b) where an amplification of the harmonics of the primary CFI mode has been reported near the FFS.

A close inspection of the thermal maps for the small $\left(A_{3}\right)$ and moderate $\left(B_{3}\right)$ FFS (figure $5 \mathrm{II} b-\mathrm{II} c$ ), reveal a non-monotonic amplification pattern which develops along the $x$-direction downstream of the FFS step edge at the wavelength of the primary CFI mode $\left(\lambda_{z} / \lambda_{z, D}=1\right)$. This non-monotonic pattern corresponds to the observations by Eppink \& Casper (2019) and Eppink (2020b) where it was found that as the CF vortices interact with the step they experience a first region of strong amplification, followed by an equally strong reduction and a second region of amplification thus creating a growth-decay-growth pattern of the $\mathrm{CF}$ vortices. The first maximum in intensity is 


\section{FFS impact on the development of crossflow instability}

located close to the step for all cases, while the first minimum and second maximum move closer to the step with increasing step height. Here, it is important to note that for cases $C_{3}$ and $D_{3}$, a different mechanism is responsible for the appearance of the second maxima. More specifically, in these cases, laminar-turbulent transition occurs within the measurement domain. The pattern of the transition reveals spanwise modulations at the same wavelength as the forced $\mathrm{CF}$ vortices. At breakdown, the increased thermal contrast between adjacent laminar and turbulent regions effectively produces the observed second maxima. Therefore, the analysis of the non-monotonic pattern is restricted exclusively to the cases $A_{3}$ and $B_{3}$ for which the boundary layer flow remains in a laminar condition for the entire measurement region. In addition, it must be stressed here that although the IR imaging has been performed in radiometric conditions (i.e. surface temperature is measured), the non-uniformity of irradiated energy from the halogen lamps as well as the varying curvature and thermal conductivity of the wing model do not allow for direct extraction of the surface heat transfer coefficient. Nevertheless, the narrow field of view of IR-A largely mitigates these effects allowing for a qualitative estimation of the amplitude of spanwise modulations in the near-wall shear around the step. In order to draw conclusions on the origin of this behaviour in the thermal maps, quantitative velocity measurements are essential, as described in the following sections.

The spatial spectral analysis employed here can be further exploited to gain insight into the organisation of the incoming CF vortices near the step. As previously discussed, the thermal maps are representative of the local magnitude of the skin friction coefficient, which in turn is related to the local velocity shear near the wall. In the cases discussed above, the flow near the step is predominantly laminar and is being actively heated. As such, regions of high temperature (i.e. brighter colour) represent low shear areas and vice versa. The characteristic streaky pattern is then qualitatively correlated with the modulation of the laminar boundary layer typical of stationary CFI. Based on the aforementioned, by unwrapping the spectral phase angle $(\phi)$ of successive (in streamwise direction) temperature profiles, a reconstruction of the trajectory $\left(d_{z}\right)$ of the coherent structures near the wing surface can be inferred.

Figure 5(I $a$ ) presents for the clean configuration a comparison of the trajectory of the $\mathrm{CF}$ vortices (solid black line) with a reference line (dashed black line) featuring a streamline angle of $\Psi^{*}=45^{\circ}$ (i.e. parallel to streamwise $X$-direction). The results indicate that the axis of the $\mathrm{CF}$ vortices is slightly tilted towards the inboard side of the wing resulting in a near-wall streamline angle of approximately $\bar{\Psi}_{w}^{*}=42.6^{\circ}$, which closely matches the one predicted by linear stability theory $\bar{\Psi}_{L S T}^{*}=41.2^{\circ}$ (dash-dotted line in figure 5I $a$ ). This behaviour is in agreement with previous experiments by Serpieri \& Kotsonis (2016) which for similar conditions showed that the inclination of the near-wall streamline differs also by a few degrees from the streamwise $X$-direction in the measurement region.

The collected trajectories for all cases are presented in figure 5(III c). As a reference for all cases, the clean configuration is given as a solid black line. Evidently, the interaction with the FFS results in a modification of the trajectory of the CF vortices given by the colour-coded lines. Figure $5(\mathrm{IV} c)$, shows the relative trajectory changes $\left(\Delta_{z}=d_{z, S I}-d_{z, C}\right)$ between the clean $\left(d_{z, C}\right)$ and the FFS $\left(d_{z, S I}\right)$ cases. For the small FFS case $\left(A_{3}\right)$, as the vortices reach the FFS, their trajectory strongly curves towards the outboard side of the wing (i.e. positive $z$ direction). Shortly downstream of the step edge, the trajectory shows a very sharp turn towards the inboard side of the wing (i.e. negative $z$ direction) before bending outboard again and relaxing to a direction almost parallel to the trajectory pertaining the clean configuration. Qualitatively, the observed behaviour is similar for all the tested FFS cases, albeit intensifying with increasing step height. 


\section{A.F. Rius-Vidales and M. Kotsonis}

A similar behaviour has been reported by Eppink (2018) when analysing the inviscid and near wall streamlines. In addition, Eppink (2018) showed that near the FFS the bending of the near-wall streamline is more pronounced than the one experienced by the inviscid streamline.

The outboard-inboard-outboard trajectory near the step can be traced to a local modification of the pressure gradient by the FFS. Numerical simulations by Tufts et al. (2017) and experimental measurements by Duncan et al. (2014a) showed that the addition of an FFS results in strong modification of the pressure gradient near the step. Hence, as the laminar boundary layer and developing CF vortices approach the step, they will first encounter an adverse pressure gradient upstream of the step, followed by a localised favourable pressure gradient at the step position and a second region of adverse pressure gradient as the flow recovers downstream of the step. The influence of the FFS on the near-step development and the modification to the structure of the $\mathrm{CF}$ vortices will be further analysed in $\S 4$.

\section{Streamwise development of CFI}

The global and local influence of the FFS on the transition behaviour and topology discussed in $\S 3$ suggest that complex flow dynamics occur when $\mathrm{CF}$ vortices interact with the FFS. This section explores the impact of an FFS on the development of the CFI by comparing and contrasting the planar-PIV measurements for the clean baseline configuration and the FFS cases as indicated on table 1.

\subsection{Impact of the FFS on the spanwise-averaged flow}

As discussed in the previous section, the numerical simulations by Tufts et al. (2017) and experimental measurements by Duncan et al. (2014a) indicate that the addition of the FFS results in a local modification of the pressure field which results in strong regions of adverse and favourable pressure gradient. In this work, limitations on the experimental set-up (figure 1) restrict the static pressure measurements to the clean configuration. Nevertheless, the effects of the pressure gradient near the step are well captured in the change of the near-wall flow trajectories (figure $5 \mathrm{III} c, \mathrm{IV} c$ ) and the streamwise evolution of the boundary layer investigated using PIV measurements of the spanwise $(w)$ and vertical $(v)$ velocity components presented in this section.

The velocity measurements are conducted in $z-y$ planes (i.e. parallel to the leading edge and normal to the wing surface, figure 1) at various $x / c_{x}$ stations. The mean flow $\bar{w}_{z}$ and $\bar{v}_{z}$ is calculated by averaging the time-average velocity components along the spanwise direction at each $x / c_{x}$ station. Note, that based on the experimental set-up (see figure 1c) a positive $\bar{w}_{z}$ value indicates an average outboard (i.e. from the root towards the tip of the wing) flow movement. For clarity, the vertical $y^{*}$-coordinate is referenced to the clean baseline model surface, while the $y$-coordinate offsets the step height at its location. In addition, the vertical coordinates are non-dimensionalised with the displacement thickness $\delta_{w, r}^{*}=514 \mu \mathrm{m}$, extracted from the $w$ spanwise velocity PIV measurements at the most upstream plane $\left(x / c_{x}=0.176\right)$ for the clean baseline case.

A comparison of the FFS cases with the clean configuration at selected stations (figure 6) reconciles the existence of an adverse pressure gradient upstream of the step which causes a deceleration in the boundary layer flow and a reduction in the spanwise $\bar{w}_{z}$ average velocity component (figure $6 \mathrm{I} b$ ). This effect intensifies as the step height increases, and results in growth of the boundary layer displacement $\left(\delta_{w}^{*}\right)$ and momentum $\left(\theta_{w}\right)$ thickness upstream of the FFS location, as shown in figure 6(c). Conversely, due to 


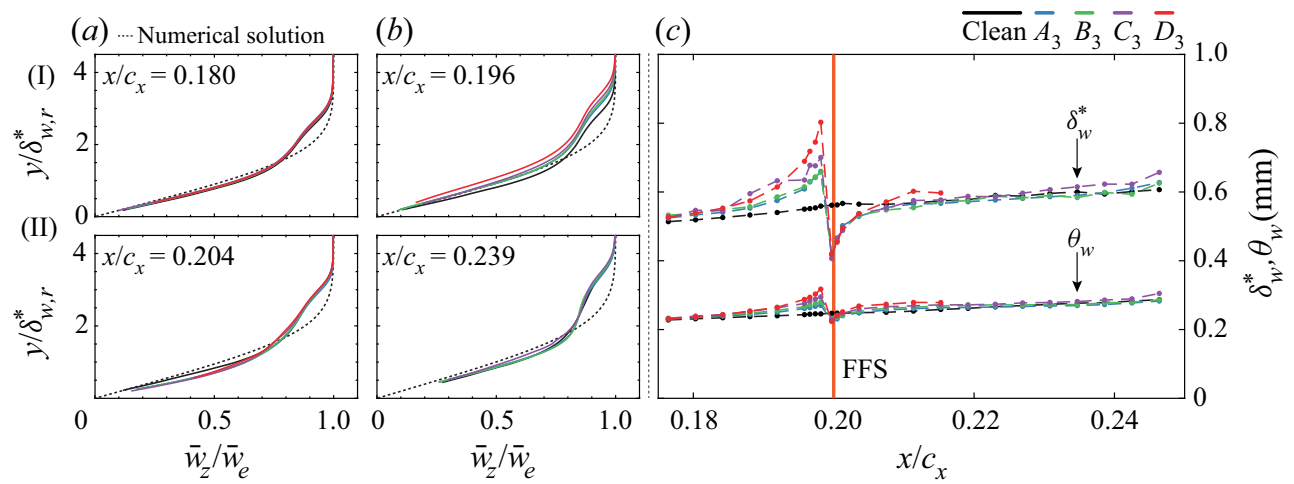

Figure 6. Selected profiles of spanwise mean flow velocity $\bar{w}_{z}(a, b)$ upstream and downstream of the FFS and $(c)$ boundary layer properties (Here $\left.\delta_{w, r}^{*}=514 \mu \mathrm{m}\right)$. The dashed black line in $(a)$ and $(b)$ denotes the numerical boundary layer solution calculated from the pressure distribution in the clean configuration.

the favourable pressure gradient downstream of the FFS edge at $x / c_{x}=0.204$ (figure 6II $a$ ) there is an acceleration of the boundary layer flow which leads to a reduction in $\delta_{w}^{*}$ and $\theta_{w}$. Farther downstream, at $x / c_{x}=0.239$ (figure 6II $b$ ), the velocity profiles collapse to the clean baseline condition except for the case $D_{3}$ (i.e. highest FFS), not shown in the figure since it transitions to turbulent flow around $x_{t} / c_{x} \approx 0.22$ (figure $3 a$ ).

Experimental observations by Eppink $(2020 b)$ in the streamline-oriented reference frame, indicate a decrease in the velocity component tangent to the streamline $\left(u_{s}\right)$ and a decrease in the CF component $\left(w_{s}\right)$ upstream of the FFS, which leads to a strong reversal of the $\mathrm{CF}$ velocity profile. These observations are in agreement with the near-wall trajectories presented in figure $5(\mathrm{III} c, \mathrm{IV} c)$, which shows that an outboard spanwise motion occurs upstream of the FFS. In the present work, it becomes evident that the face of the FFS forms the equivalent of an attachment line. As expected, due to the sharp FFS geometry near the step location, the incoming boundary layer experiences a deceleration in $\bar{u}_{z}$. Although no surface irregularity or pressure gradient can form in the $z$ direction, the modification of $\bar{u}_{z}$ directly couples to changes of $\bar{w}_{z}$, through momentum coupling. As such, a strong conversion of wall tangent velocity components (explicitly $\bar{u}_{z}$ and by consequence $\bar{w}_{z}$ ) into a wall normal velocity component $\left(\bar{v}_{z}\right)$ is occurring. Evidence of this behaviour is shown in figure 7(I $a-\mathrm{II} e$ ) in which all FFS cases lead to a considerable increase in the spanwise-averaged time-averaged vertical velocity $\bar{v}_{z}$ at the step. For the highest FFS case $D_{3}$, the maximum vertical velocity reaches a value of approximately 10 per cent of the external spanwise velocity. These results are in agreement with Eppink (2020b) where similar levels of amplification are reported. This rigorous upward flow movement by the FFS which affects the boundary layer flow will be analysed in more detail on the following sections.

\subsection{Impact of the FFS on the time-averaged total flow}

As elaborated in $\$ 2.1$, the investigation of the influence of an FFS on the development and breakdown of CFI is facilitated using DREs near the leading edge to condition the wavelength and amplitude of the disturbances. This conditioning allows for a highly periodic and uniform amplitude distribution for the ensuing $\mathrm{CF}$ vortices (figure 5), which further facilitates the extraction of pertinent information from the velocity fields in terms of spanwise spectral modes. 


\section{A.F. Rius-Vidales and M. Kotsonis}

\section{$\square$ Model surface $\square$ FFS $\square$ No data}

(a)

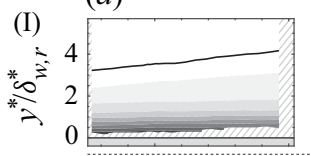

(II)
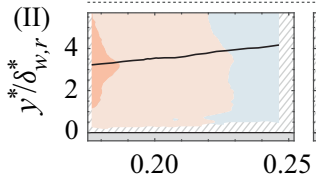

$x / c_{x}$ (b)
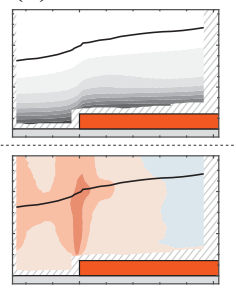

$0.20 \quad 0.25$ (c)

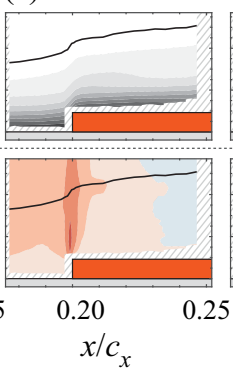

(d)

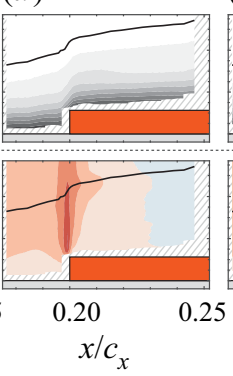

(e)

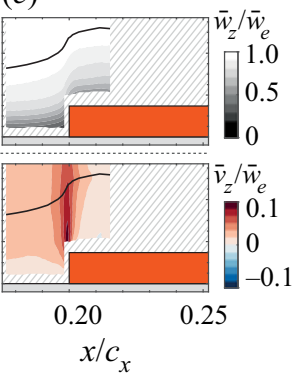

Figure 7. Contours of spanwise mean flow velocity (flow direction from left to right) $\bar{w}_{z}$ (I) and $\bar{v}_{z}$ (II): (a) clean; (b) $A_{3} ;(c) B_{3} ;(d) C_{3} ;(e) D_{3}$. The solid black line denotes $\delta_{99, w}\left(\right.$ Here $\left.\delta_{w, r}^{*}=514 \mu \mathrm{m}\right)$.

More specifically, for the analysis of the PIV measurements, the time-average and standard deviation of the velocity components have been spatially filtered using Fourier transformations. As indicated on the diagram in figure 8, at each wall-normal position in the measurement plane, a fast Fourier transform (FFT) has been applied on extracted velocity profiles along the $z$-direction. Subsequently, a pertinent set of Fourier modes are selected to reconstruct the velocity through the inverse fast Fourier transform (IFFT). In addition to enhancing the velocity fields by reducing the measurement noise (which typically appears at small wavelengths), this technique offers the possibility to isolate the effect of the FFS on a particular harmonic of the CFI mode. Hence, for the remainder of the analysis, the reconstructed velocity fields (subscript $R$ ) are used unless otherwise noted. Moreover, the notation $m(0, n)$ (Bippes 1999; Wassermann \& Kloker 2002) is used to indicate the Fourier modes used during the reconstruction. Note that the first index in parenthesis corresponds to the frequency of the mode, which for the present study is zero, and indicates a stationary CFI mode while the second index (n) denotes multiples of the spanwise wavenumber of the CF vortices forced by the DRE. In addition, when indicated by the summation convention $\left(\sum_{n}^{5} m(0, n)\right)$, the velocity fields have been partially reconstructed using the wavelengths contained between the primary force CFI mode $\left(\lambda_{z, D}=7.5 \mathrm{~mm}\right)$ and its fifth harmonic $\left(\lambda_{z, D}=1.5 \mathrm{~mm}\right)$.

Figures 9 and 10 show the reconstructed fields for the spanwise $\left(\bar{w}_{R}\right)$ and vertical $\left(\bar{v}_{R}\right)$ time-average velocity, respectively, for the clean and FFS cases at selected locations upstream and downstream of the step. Note that the $z^{*}$-coordinate features a shift with respect to the $z$-coordinate origin such that the $\mathrm{CF}$ vortices align between presented streamwise stations. In the clean configuration, the spanwise velocity contours $\bar{w}_{R}$ presented in figure $9(\mathrm{I} a)$, show a pair of stationary corotating vortices. These stationary vortices are evenly spaced at the forced wavelength $\left(\lambda_{z, D}=7.5 \mathrm{~mm}\right)$ for the entire measurement region as shown by the spatial spectral analysis on the thermal maps presented in figure 5(II $a)$. These $\mathrm{CF}$ vortices develop inside the boundary layer, where they transfer high momentum flow towards the wall (downwelling region, $\oplus$ in figure $9 \mathrm{I} a$ ) and low momentum flow away from it (upwelling region, $\ominus$ in figure 9I $a$ ). As these structures evolve downstream, their amplitude and inherent distortion they impart on the mean flow increases (Wassermann \& Kloker 2002; White \& Saric 2005; Downs \& White 2013). For the condition under study, the initial amplitude provided by the DREs results in a set of nonlinearly amplified vortices when reaching the step location. 


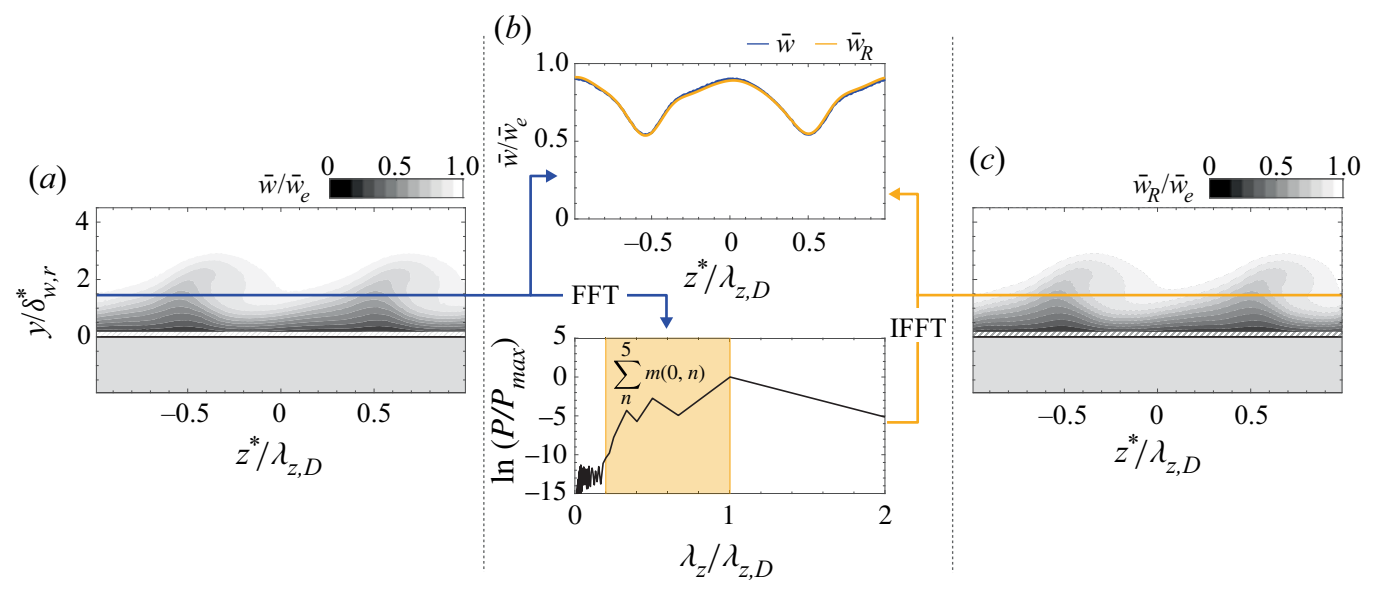

Figure 8. Schematic of spatial filtering of time-averaged spanwise velocity $w$ contours (10 levels from 0 to 1 , $z^{*}$ positive direction outboard) for the clean case: $(a)$ original velocity field; $(b)$ spectral decomposition; (c) reconstructed velocity field.

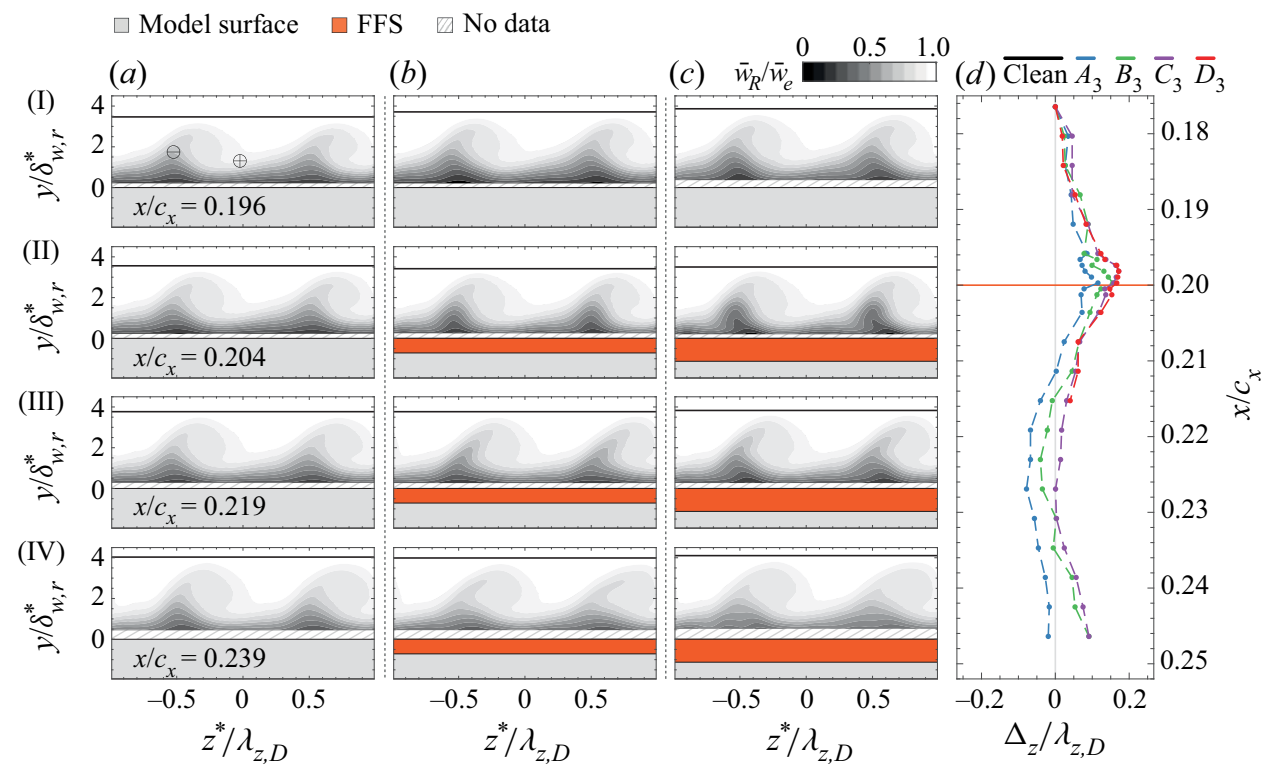

Figure 9. Contours of time-averaged spanwise velocity ( $z^{*}$ positive direction outboard), spatially filtered between $\sum_{n}^{5} m(0, n)$ : (a) clean; $(b) A_{3} ;(c) C_{3} ;(d)$ change in trajectory of the CF vortices due to the FFS. The solid black line denotes $\delta_{99, w}\left(\right.$ Here $\delta_{w, r}^{*}=514 \mu \mathrm{m}$ and $\left.\lambda_{z, D}=7.5 \mu \mathrm{m}\right)$.

The interaction of the stationary CF vortices with the FFS results in topological modifications within the boundary layer as shown in figure 9 . As the CF vortices reach the FFS $\left(x / c_{x}=0.196\right.$, figure $\left.9 \mathrm{I} b-\mathrm{I} c\right)$ there is an evident increase of the transfer of low momentum flow away from the wall (i.e. upwelling region). This corresponds well with the general upward deflection and streamwise deceleration of the incoming flow evident in figures 6 and 7, as well as the observations of Eppink (2020b) which indicate an amplification of the vertical velocity component near the FFS. At the same streamwise location, the vertical velocity contours $\left(\bar{v}_{R}\right)$ in figure $10(\mathrm{I} b-\mathrm{I} c)$ indicate a strong increase, 


\section{A.F. Rius-Vidales and M. Kotsonis}

$\square$ Model surface $\square$ FFS $\square$ No data

(a)

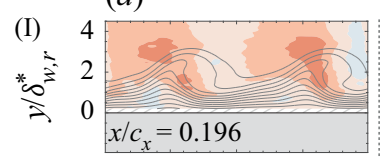

\begin{tabular}{ll|l|} 
(II) & 4 \\
*a & 2
\end{tabular}

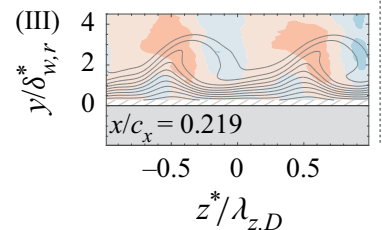

(b)
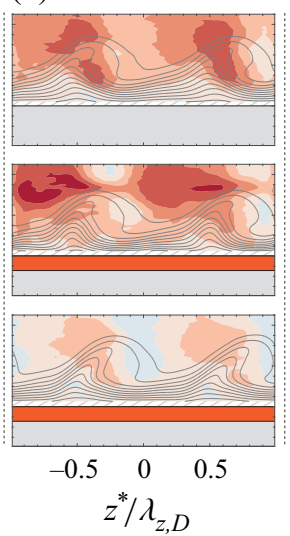

(c)
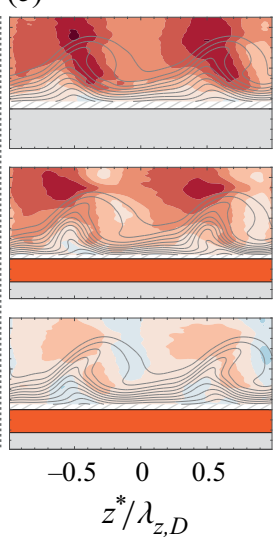

(d)
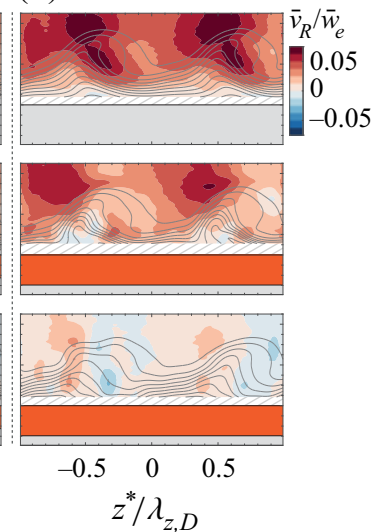

Figure 10. Contours of time-averaged vertical velocity and spanwise velocity (grey line 10 levels from 0 to 1 ) $z$ positive direction outboard, spatially filtered between $\sum_{n}^{5} m(0, n):(a)$ clean; (b) $A_{3} ;(c) C_{3} ;(d) D_{3}$. (Here $\delta_{w, r}^{*}=514 \mu \mathrm{m}$ and $\left.\lambda_{z, D}=7.5 \mu \mathrm{m}\right)$.

especially on the inner section of the upwelling region. Henceforth, the overall increase of vertical velocity at the step location is suggestive of the underlying amplification of the instabilities as a result of the flow modifications incurred by the step.

The near-wall structures trajectory identified in $\S 3$ revealed an outboard-inboardoutboard motion near the wall. In contrast to IR imaging, the PIV measurements facilitate the inspection of this flow deflection away from the wall. Specifically, profiles of spanwise velocity perturbation $\left(\hat{w}_{R}\right)$ are extracted along the $z$-direction at the height of the stationary disturbance profile maxima (calculated as described in § 2.3) for each measurement plane. Successive spanwise profiles are compared using a vector convolution (i.e. cross-correlation). The result of this analysis is referenced to the most upstream measurement plane and yields the relative location $\left(d_{z}\right)$ along the $z$-direction assumed by the CF vortices as they evolve downstream. Figure $9(d)$ shows the relative change of trajectory $\left(\Delta_{z}=\mathrm{d} z_{S I}-\mathrm{d} z_{C}\right)$ between the FFS cases $\left(\mathrm{d} z_{S I}\right)$ and the clean configuration $\left(\mathrm{d} z_{C}\right)$, confirming that the outboard-inboard-outboard motion described by the near-wall trajectory also occurs away from the wall, in proportional intensity to the FFS height.

Eppink (2018) reported an abrupt change of the near-wall streamline angle as the boundary layer flow intercepts the FFS. Henceforth, to evaluate the spanwise motion effect as a function of the distance from the wall, figure 11( $\mathrm{I} a-\mathrm{II} b)$ shows a set of wall-parallel $\overline{w_{R}}$ and $\overline{v_{R}}$ planes extracted away from the wall $(400 \mu \mathrm{m}$ above the height of the stationary disturbance profile maxima). The dashed lines in figure 11(c) indicate the relative shift $\left(\Delta_{z}\right)$ along the $z$ direction calculated for each configuration. Additionally, wall-parallel planes extracted at a vertical location closer to the wall $(200 \mu \mathrm{m}$ below the height of the stationary disturbance profile maxima) are presented in figure $11(\mathrm{III} a-\mathrm{IV} b)$ with $\Delta_{z}$ in figure $11(d)$. A comparison of the $\Delta_{z}$ above and below (figure $11 c, d$ ) the stationary disturbance profile maxima confirm that the outboard-inboard-outboard motion intensifies closer to the wall.

Summarising up to this point, the observed influence of the FFS on the developing boundary layer reveal topological changes in both spanwise averaged flow as well as stationary $\mathrm{CF}$ vortices. As the incoming flow approaches the step, a strong 
- FFS 四 No data

Clean $\bar{A}_{3} \bar{B}_{3} \bar{C}_{3} \bar{D}_{3}$

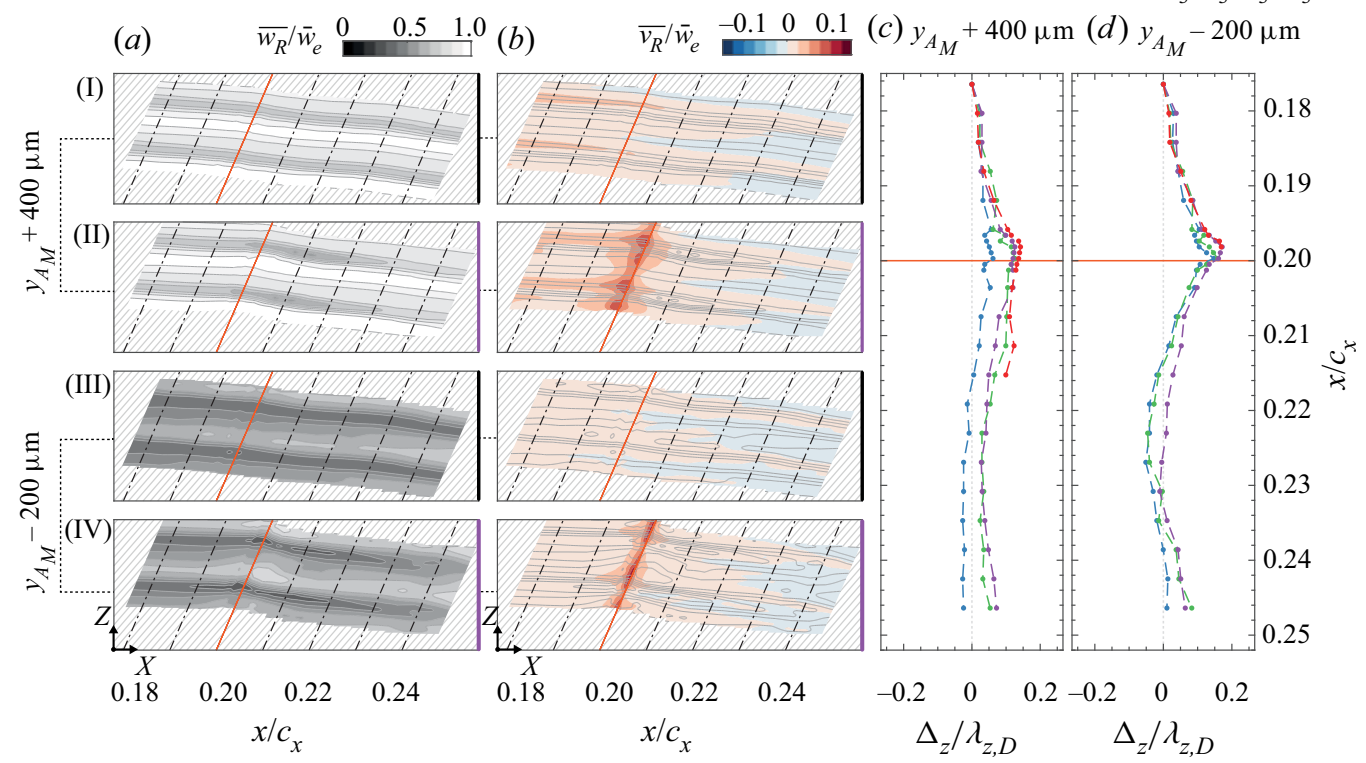

Figure 11. Wall-parallel contour maps of time-averaged spanwise and vertical velocity spatially filtered between $\sum_{n}^{5} m(0, n)$. The contours in rows I and II are extracted at $400 \mu \mathrm{m}$ above the height of the steady perturbation maxima $\left(y_{A_{M}}\right)$, while the contours in rows III and IV are extracted closer to the wall at $200 \mu \mathrm{m}$ below $y_{A_{M}}$ : (I,III) clean case; (II,IV) $C_{3}$ case; and panels $(c, d)$ the corresponding relative change of trajectory $\left(\Delta_{z}=\mathrm{d} z_{S I}-\mathrm{d} z_{C}\right)$.

outboard-inboard-outboard spanwise motion of the CF vortices is evident. This reflects the non-monotonic changes in pressure imposed by the step (Duncan et al. 2014a; Tufts et al. 2017). This effect is stronger near the wall, where the deceleration in the base flow is larger. Consequentially, an intense ejection of vertical velocity is documented at the vicinity of the step in agreement with Eppink (2020b), resulting from the upward deflection of incoming wall-tangent flow as the latter engages with the step. The role of the spanwise motion on the overall transition scenario cannot be conclusively inferred by the presented measurements. However, potential candidate mechanisms can be proposed. A simple interpretation of this motion can be traced simply on the underlying changes of the base flow, which in turn can lead to modifications of the wavenumber vector of an incoming instability. A different effect could be the potential appearance of non-modal effects, in a mechanism similar to the well known lift-up effect (Landahl 1980; Marxen et al. 2009; Brandt 2014) active in regions of strong shear changes. Notwithstanding the active mechanism, the relation between the outboard-inboard-outboard motion and the growth of the instability remains a point of interest. To elucidate this, fully three-dimensional velocity measurements or detailed numerical simulations of these flows are deemed necessary.

\subsection{Impact of the FFS on the primary stationary CFI and harmonics}

As described in $\S 2.3$, the experimentally measured mode-shape of the stationary disturbance profile is equivalent to the spanwise root mean square $\left(\langle\rangle_{z}\right)$ of the time-average perturbation $\left(\hat{w}_{R}\right.$ or $\left.\hat{v}_{R}\right)$. Previous experimental studies on CFI (White \& Saric 2005; Downs \& White 2013; Serpieri \& Kotsonis 2016) have used this metric in the analysis 


\section{A.F. Rius-Vidales and M. Kotsonis}

$\square$ Model surface $\square$ FFS $\square$ No data

(a)

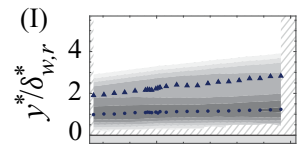

(II)

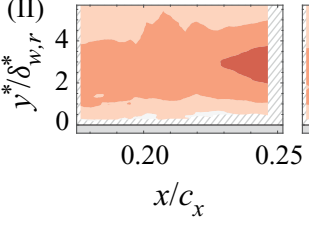

(b)
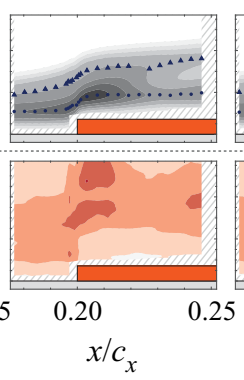

(c)
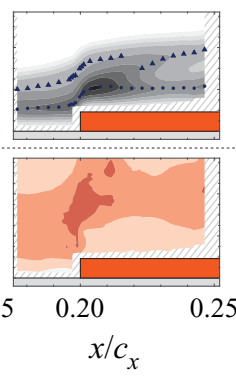

(d)

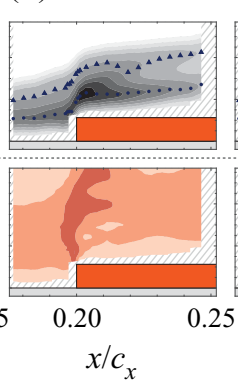

(e)

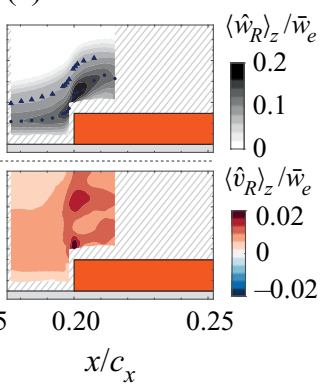

Figure 12. Contours of steady disturbances profiles (flow direction from left to right) $\left\langle\hat{w}_{R}\right\rangle_{z}$ (I) and $\left\langle\hat{v}_{R}\right\rangle_{z}$ (II) spatially filtered between $\sum_{n}^{5} m(0, n)$. Location of lower $(\bullet)$ and upper lobe $(\boldsymbol{\Delta})$ : $(a)$ clean; $(b) A_{3} ;(c) B_{3}$; (d) $C_{3} ;(e) D_{3}$. (Here $\left.\delta_{w, r}^{*}=514 \mu \mathrm{m}\right)$.

of the Euclidean sum of the vertical and streamwise velocity components measured by hot-wire anemometers. By merit of the chosen imaging planes in the present study, the disturbance profiles have been calculated independently for each measured velocity component.

Figure 12 presents contour plots of the stationary disturbance profiles $\left\langle\hat{w}_{R}\right\rangle_{z}$ and $\left\langle\hat{v}_{R}\right\rangle_{z}$ calculated on the partial reconstructed fields (i.e. $\left.\sum_{n}^{5} m(0, n)\right)$ for all FFS cases. The results for the spanwise disturbance profiles $\left(\left\langle\hat{w}_{R}\right\rangle_{z}\right)$ in figure 12(I $\left.a-\mathrm{I} e\right)$ show a distinguishable lower lobe upstream of the FFS at $x / c_{x}=0.18$, situated near the wall at $y / \delta_{w, r}^{*} \approx 1$, which corresponds to the wall-normal maxima of these profiles (• markers in figure 12I $a-\mathrm{I} d$ ). In addition, a second upper lobe is also identified ( $\Delta$ markers in figure 12I $a-\mathrm{I} d$ ). The appearance of this feature has been linked to the mean flow distortion typical in the nonlinear stages of stationary CFI development (Haynes \& Reed 2000; White \& Saric 2005). Near the step location at $x / c_{x}=0.2$ there is an evident amplification of the spanwise disturbance profiles $\left(\left\langle\hat{w}_{R}\right\rangle_{z}\right)$ followed by a decay $\left(x / c_{x}>0.21\right)$ in all measured cases. Moreover, for $x / c_{x}>0.22$ the second upper lobe ( $\boldsymbol{\Delta}$ markers in figure 12I $a-\mathrm{I} d$ ) becomes increasingly evident, indicating a strong mean flow distortion as shown in figure $9(\mathrm{IV} a-\mathrm{IV} c)$.

Previous studies (Tufts et al. 2017; Cooke et al. 2019; Eppink 2020b) have reported the development of a strong peak near the wall in the disturbance profiles downstream of the step location (see figure 10 in Eppink (2020b) and figure A5a in Tufts et al. (2017)). In this work, a second peak near the wall is not evident in the spanwise perturbation profiles $\left\langle\hat{w}_{R}\right\rangle_{z}$, since as shown in figure 12 ( $\left.\mathrm{I} a-\mathrm{I} e\right)$ only one lower lobe is present upstream and downstream of the FFS for all the configurations. The lack of the near-wall structure in the present study can be attributed to unresolved regions in the PIV planes, originating from wall reflections. Nevertheless, the vertical perturbation profiles $\left\langle\hat{v}_{R}\right\rangle_{z}$ (figure $12 \mathrm{II} a-\mathrm{II} d$ ) for the highest step case $D_{3}$ do reveal a clear peak near the wall at the location of the FFS in agreement with Eppink (2020b).

Figure 13 presents the spanwise disturbance $\left\langle\hat{w}_{R}\right\rangle_{z}$ profiles extracted at selected streamwise locations upstream and downstream of the FFS for the partial reconstructed field $\left(\sum_{n}^{5} m(0, n)\right.$, figure 13I $\left.a-\mathrm{IV} a\right)$, primary forced mode $(m(0,1)$, figure $13 \mathrm{I} b-\mathrm{IV} b)$ and first harmonic $(m(0,2)$, figure $13 \mathrm{I} c-\mathrm{IV} c)$. In all FFS cases there is a considerable upstream effect which leads to a substantial increase in the profiles maxima $\left(\max \left(\left\langle\hat{w}_{R}\right\rangle_{z}\right)\right.$, 


\section{$\overline{\text { Clean }} \bar{A}_{3} \bar{B}_{3} \bar{C}_{3} \bar{D}_{3}$}

(a)
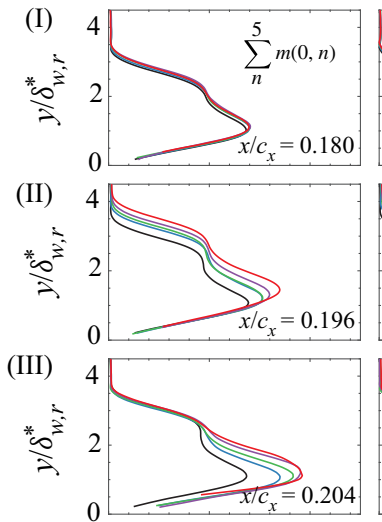

(IV)

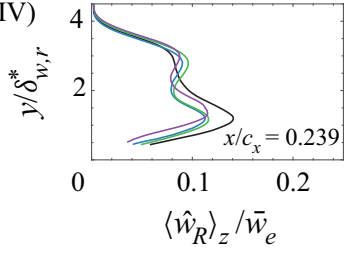

(b)
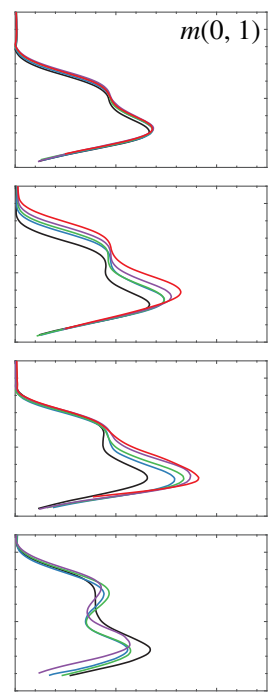

$0.1 \quad 0.2$

$\left\langle\hat{w}_{R}\right\rangle_{z} / \bar{w}_{e}$ (c)
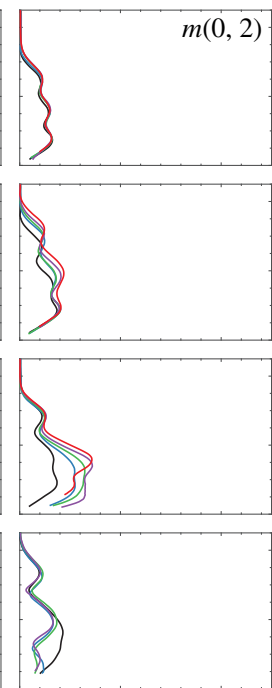

$0 \quad 0$ (d)

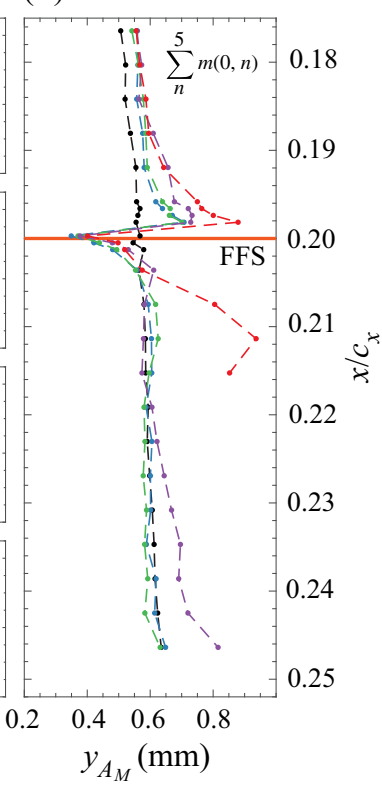

Figure 13. Selected profiles $(a-c)$ of the steady disturbance $\left\langle\hat{w}_{R}\right\rangle_{z}$ upstream and downstream of the FFS for the primary CFI modes and its first harmonic. (d) Streamwise evolution of the steady disturbance maxima wall-normal height $\left(y_{A_{M}}\right)\left(\right.$ Here $\left.\delta_{w, r}^{*}=514 \mu \mathrm{m}\right)$.

figure 13II $a$ ). It is noteworthy that the amplitude increase coincides with the localised increase in the vertical velocity component $(v)$, the region of outboard spanwise motion of the $\mathrm{CF}$ vortices (figures $9 d$ and $5 \mathrm{IV} c$ ) and possible reversal of the CF velocity component (Eppink 2020b, figures 8 and 9).

Figure $13(d)$, shows the streamwise evolution of the wall-normal location of the lower lobe (i.e. closer to the wall) maxima $\left(y_{A_{M}}\right)$ of the spanwise steady disturbance profiles $\left\langle\hat{w}_{R}\right\rangle_{z}$ for the partial reconstructed field. Note that the $y$-coordinate reference is the model surface and therefore it has been offset by the step height. In agreement with Eppink $(2018,2020 b)$ as the CF vortices approach the step they experience an increase in $y_{A_{M}}$, essentially lifting off the surface before reaching the FFS edge. Just downstream of the step edge $\left(x / c_{x}=0.204\right.$, figure $\left.13 \mathrm{III} a\right)$ there is a decrease in $y_{A_{M}}$ as there is a reduction in the boundary layer displacement thickness $\left(\delta_{w}^{*}\right.$ in figure $6 c$ ) and a sudden sharp increase in the maximum amplitude of the primary stationary disturbance $(m(0,1)$, figure $13 \mathrm{III} b)$. The highest step height $\left(D_{3}\right)$ is responsible for the maximum amplification. Similarly, the $\left\langle\hat{w}_{R}\right\rangle_{z}$ profiles corresponding to the reconstructions using only the first harmonic $(m(0,2)$, figure $13 \mathrm{I} c-\mathrm{IV} c$ ) show a considerable amplification at the step location.

In addition to changes in disturbance amplitude, the strong amplification of the primary disturbance mode $m(0,1)$ near the FFS edge results in a significant mean flow distortion and saturation of the CF vortices. This mean flow distortion is evident in the change of the shape of the mode which leads to the development of two clearly distinguishable local maxima, as shown in figure 13(IVb). In a smooth wing CFI development, the appearance of this second upper lobe signals a typical nonlinear development of stationary CFI which results in a strong mean flow distortion and the onset of secondary instability modes (Haynes \& Reed 2000; White \& Saric 2005). Nevertheless, in this case, breakdown of 


\section{A.F. Rius-Vidales and M. Kotsonis}

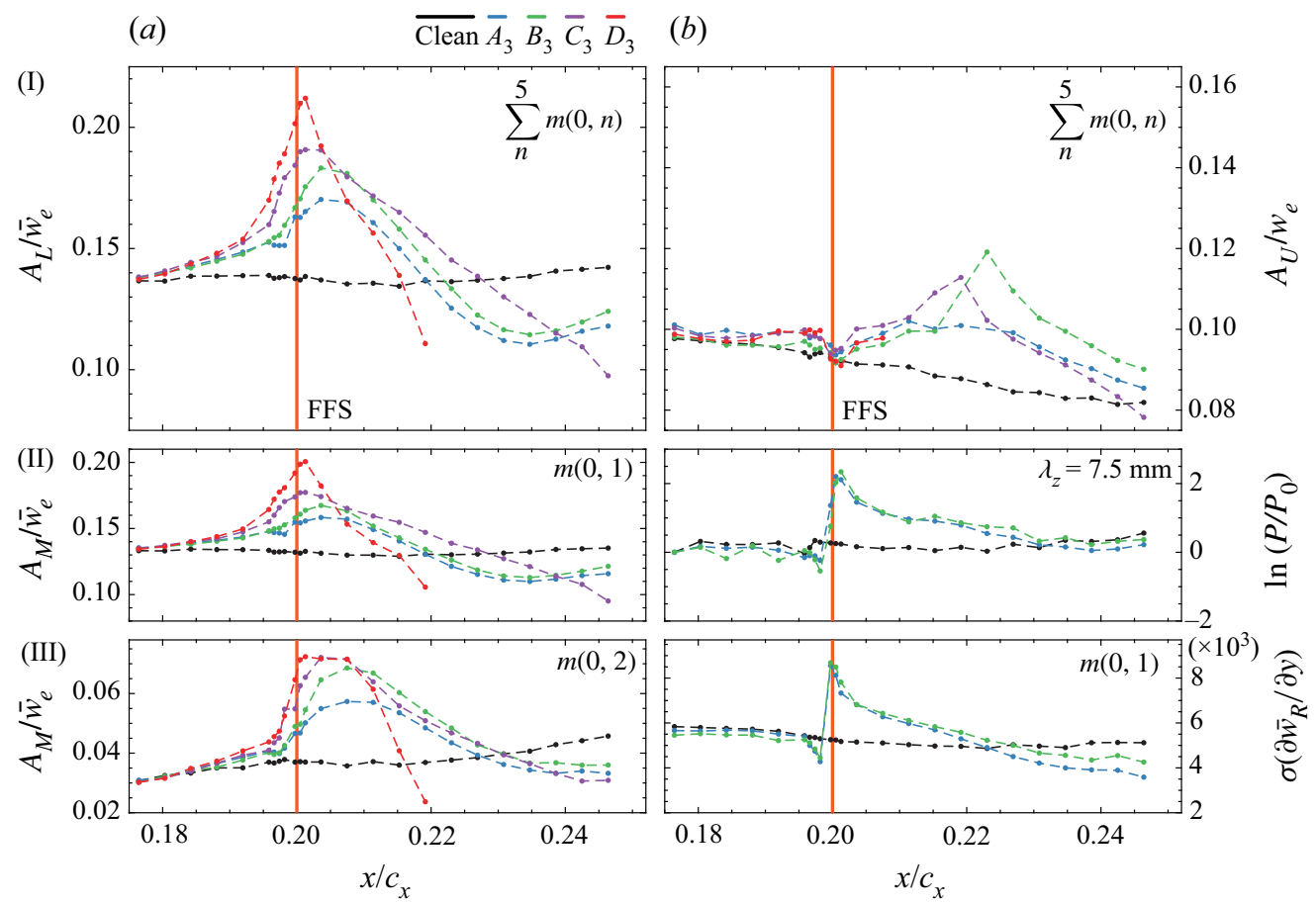

Figure 14. Steady disturbance profile amplitudes: (I $a$ ) lower lobe amplitude $\left(A_{L}\right)$; $(\mathrm{I} b)$ upper lobe amplitude $\left(A_{U}\right)$; (II $\left.a\right)$ primary CFI mode $(m(0,1))$ maximum amplitude; (II $\left.a\right)$ first harmonic $(m(0,2))$ maximum amplitude; (II $b)$ IR-B PSD extracted at $\lambda_{z}=7.5 \mu \mathrm{m}$; (III $\left.b\right)$ primary CFI mode $(m(0,1))$ standard deviation along the span direction of the wall-normal gradient at $y / \delta_{w, r}^{*}=0.68$.

the CF vortices only occurs for the case $C_{3}$ at $x_{t} / c_{x} \approx 0.27$ and $D_{3}$ at $x_{t} / c_{x} \approx 0.22$ as indicated on figure $3(a)$.

Based on the partial reconstruction (i.e. $\left.\sum_{n}^{5} m(0, n)\right)$, the amplitudes of the disturbance profile $\left\langle\hat{w}_{R}\right\rangle_{z}$ lower $\left(A_{L}\right)$ and upper $\left(A_{U}\right)$ lobes are presented in figure $14(\mathrm{I} a-\mathrm{I} b)$. The results for the FFS cases indicate that the amplitude of the lower lobe $\left(A_{L}\right)$ intensifies rapidly upstream of the step and reaches a maximum value just downstream of the step edge. Hereinafter, as the amplitude of the upper lobe increases $\left(A_{U}\right)$ (i.e. stronger mean flow distortion), there is a steep decay in $A_{L}$ which leads to values below the clean baseline case (black line in figure $14 a$ ). Once this minimum is reached a second region of growth is observed for the cases $A_{3}$ and $B_{3}$, which do not undergo laminar breakdown in the region $0.18 \leqslant x / c_{x} \leqslant 0.25$ as indicated by the IR thermal maps on figure $5(\mathrm{I} b-\mathrm{I} c)$.

The overall amplification trend is in good agreement with the detailed experimental work presented by Eppink \& Casper (2019) and Eppink (2020b), where a similar amplification-decay-amplification pattern has been observed on the $\langle\hat{u}\rangle_{z}$ perturbation peak amplitude. Moreover, the results presented in figure 14 in Eppink (2020b) indicate that the slope of the second region of growth becomes steeper with increasing step height.

Evidence of this amplification-decay-amplification pattern was previously presented in the spectra of the IR thermal maps (figure 5) for the small $\left(A_{3}\right)$ and moderate $\left(B_{3}\right)$ cases. As noted before, for cases $C_{3}$ and $D_{3}$, the second amplification maxima in the IR maps are instead related to the laminar-turbulent transition front. Therefore, the analysis of this pattern is restricted to the cases $A_{3}$ and $B_{3}$ for which the boundary layer flow remains in a laminar condition for the entire measurement region. To further 


\section{FFS impact on the development of crossflow instability}

probe the correlation between the growth of the $\mathrm{CF}$ vortices and the pattern observed in the spectral analysis of IR-A thermal maps, the power content $(P)$ pertaining to the primary forced mode $\left(\lambda_{z} / \lambda_{z, D}=1\right)$ is shown in figure 14(II $\left.b\right)$. At each streamwise location $P$ is normalised with the value corresponding to the most upstream $\left(x / c_{x}=\right.$ $0.176)$ location $\left(P_{0}\right)$. In addition, figure $14(\mathrm{III} b)$ presents the standard deviation along the span direction of the velocity gradient in $y\left(\partial \overline{w_{R}} / \partial y\right)$ at $y / \delta_{w, r}^{*}=0.68$ above the wall. The results presented in figure $14(\mathrm{II} b, \mathrm{III} b)$ show a striking correlation, reconciling the amplification-decay-amplification pattern in the spectra of the IR thermal maps to local changes in the surface heat transfer properties due to variations in wall shear (i.e. changes in $\partial \overline{w_{R}} / \partial y$ ) related to the amplification of the CFI by the step.

Figure 14(III $a)$ suggests a similar amplification trend for the harmonic $(m(0,2))$ past the step location, displaying a similar amplification-decay-amplification pattern. These results are in agreement with previous observations (Eppink 2020b; Rius-Vidales \& Kotsonis 2020) and support the spatial analysis of the IR measurements in figure 5, which showed an amplification of the first harmonic at the step location for all the FFS cases. However, it is striking to note the relatively 'delayed' amplification of this first harmonic compared with the primary mode. For each FFS case, the peak of $m(0,2)$ occurs downstream of the corresponding peak of $m(0,1)$. This behaviour further suggests a (partially) indirect influence of the higher harmonics by the FFS. More specifically, the primary CFI increases in amplitude due to interaction with the FFS, inherently forcing nonlinear amplification of higher harmonics as well as of mean flow distortion. In addition, future fully three-dimensional measurements in this region are deemed necessary to characterise a possible deformation of the recirculation region downstream of the FFS location.

Eppink (2020b) proposed that the first region of growth is linearly caused by a destabilisation of the stationary CFI modes due to the strong inflectional profiles caused by the adverse pressure gradient near the step. In turn, the second region is attributed to a nonlinear development due to the modulation of the recirculation region downstream of the FFS edge, which resulted in streamwise oriented vortices which amplify the harmonics of the primary mode. The behaviours observed by Eppink (2020b) and the ones explained in this work, highlight the sensitivity and complexity of the FFS-CFI interaction.

Based on the findings presented so far, the steady interaction of the CF vortices with the FFS can be summarised as follows. As the CF vortices travel towards the FFS they experience an adverse pressure gradient which results in an outboard spanwise motion (figures $5 \mathrm{IV} c$ and $9 d$ ), a decrease in the wall-tangent velocity components (figure 6I $b$ ) and an increase in the amplitude of the spanwise $(w)$ disturbance profile (figure 13II $a$ ) when compared with the clean configuration. In addition, at the step a portion of the wall-tangent velocity component converts into a strong vertical $(v)$ velocity component due to the upward deflection (figure 7II $a-\mathrm{II} e$ ). This vertical component is very localised and appears to reach a maximum on the inner side of the upwelling region of the $\mathrm{CF}$ vortices. Downstream of the step, there is a further amplification of the spanwise $(w)$ disturbance profile (figure 14I $a$ at $x / c_{x} \approx 0.205$ ) and a sudden inboard spanwise motion (i.e. favourable pressure gradient). This strong amplification drives a further increase of mean flow distortion and rise of nonlinear interactions (figures 9 and 14). This leads to a saturation of the primary $\mathrm{CF}$ vortices (figure $14 \mathrm{I} a$ at $x / c_{x} \approx 0.21$ ), and subsequent increase in harmonic amplitude. Farther downstream, the stationary CFI decay and the CF vortices experience an outboard spanwise motion (i.e. adverse pressure gradient). Finally, a second region of growth enhanced by the nominal favourable pressure distribution of the wing develops for the smaller FFS cases. 


\section{A.F. Rius-Vidales and M. Kotsonis}

One of the most significant outcomes of the aforementioned observations is the apparent mismatch between the transition location behaviour evaluated using the IR imaging maps (figure 3) and the amplitude growth of the stationary CFI as evaluated in figure 14. In fact, for all cases of FFS investigated in this work, the partial reconstructed $\left(\sum_{n}^{5} m(0, n)\right)$ amplitude at the downstream end of the PIV domain is lower than the clean case. This is certainly expected for the two highest step case $\left(C_{3}\right.$ and $\left.D_{3}\right)$, as the imminent breakdown of the vortices downstream of the step effectively breaks the spanwise modulation, 'smoothening out' the apparent amplitude. However, for the two smaller step cases $\left(A_{3}\right.$ and $B_{3}$ ), the laminar boundary layer survives the passage over the step and emerges apparently stabilised in the downstream vicinity of the step. Naturally this effect is localised, as the $\mathrm{CF}$ vortices start growing again, as evident in figure $14(\mathrm{I} a)$ as well as from the appearance of the second maxima in the thermal maps spectra (figure $5 \mathrm{II} b, \mathrm{II} c$ ). In addition, figure 6 confirms the spanwise averaged flow recovery to the clean case at $x / c_{x} \approx 0.24$. In combination, it becomes apparent that the drastic effects of the FFS on the transition location cannot be traced solely to the evolution of stationary instabilities, highlighting the importance of temporal fluctuations. Henceforth, the following discussion focuses on the effect of the FFS on the unsteady disturbances.

\subsection{Impact of the FFS on the unsteady disturbances}

The analysis of temporal velocity fluctuations in specific regions within the CF vortices provides important information regarding steady and unsteady instability development and eventual laminar breakdown. Within the highly distorted boundary layer subject to primary stationary CFI, several regions of coherent fluctuations have been identified, typically corresponding to either primary travelling modes or secondary high-frequency instabilities of Kelvin-Helmholtz type. The first region of fluctuations has been classified as a type-I mode (Wassermann \& Kloker 2002, 2003) or z-mode (Malik et al. 1999). This region coincides with the local minimum of the spanwise gradient located at the outer side of the upwelling region of the CF vortices (area $B$ in figure $15 \mathrm{III} a$ ). The second region of fluctuations has been classified as type-II mode (Wassermann \& Kloker 2002, 2003) or $y$-mode (Malik et al. 1999). This region coincides with higher levels of the wall-normal gradient and manifests near the top of the $\mathrm{CF}$ vortices (area $C$ in figure 15I $a$ ). Finally, the third region of fluctuations known as type-III mode (Wassermann \& Kloker 2002; Bonfigli \& Kloker 2007) has been observed near the wall on the inner side of the upwelling region and coincides with the local maxima of the spanwise gradient (area $A$ in figure 15III $a$ ).

The origin of the velocity fluctuations in the region pertaining to type III has been traced to the interaction between travelling and stationary CFI modes (Wassermann \& Kloker 2002, 2003; White \& Saric 2005; Serpieri \& Kotsonis 2016). The type III instability can be considered as a primary CF instability with non-zero frequency. Nevertheless, in the environment of enhanced stationary CFI modes relevant to the present study, type III instability is identified through the 'footprint' of the nonlinear interaction between stationary and travelling modes.

Conversely, the velocity fluctuations in the regions pertaining to type-I and type-II differ entirely in nature from the type-III, as they have been associated with secondary instabilities which emerge on the strong velocity shears as the $\mathrm{CF}$ vortices reach saturation. These high frequency, rapidly amplifying instabilities are extremely sensitive to small changes in the developing shears and lead to the breakdown of the CF vortices and transition to turbulent flow (Wassermann \& Kloker 2002; White \& Saric 2005; Serpieri \& Kotsonis 2016). Previous studies on the mechanisms of these instabilities have shown the 
$\square$ Model surface $\square$ FFS $\square$ No data

(a)

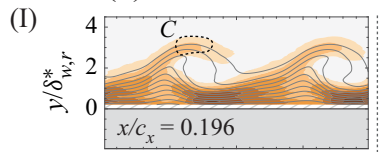

(II)
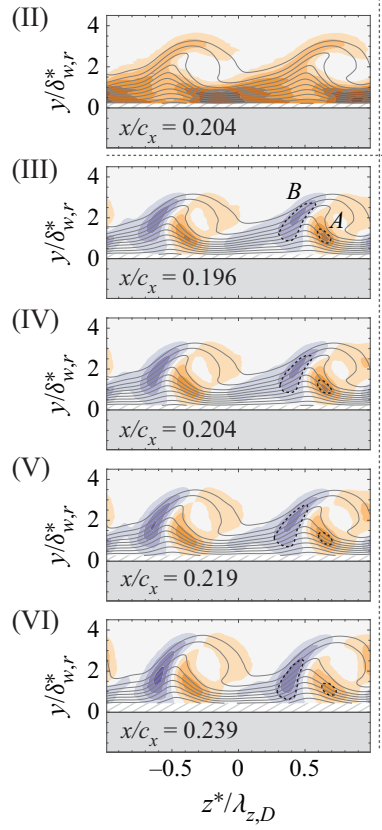

(b)
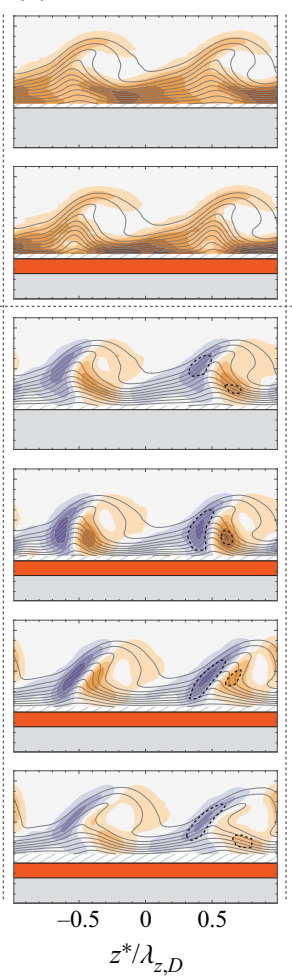

(c)
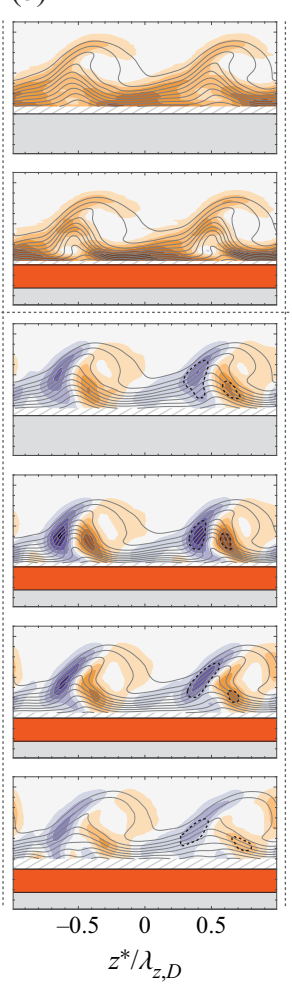

$(d)$

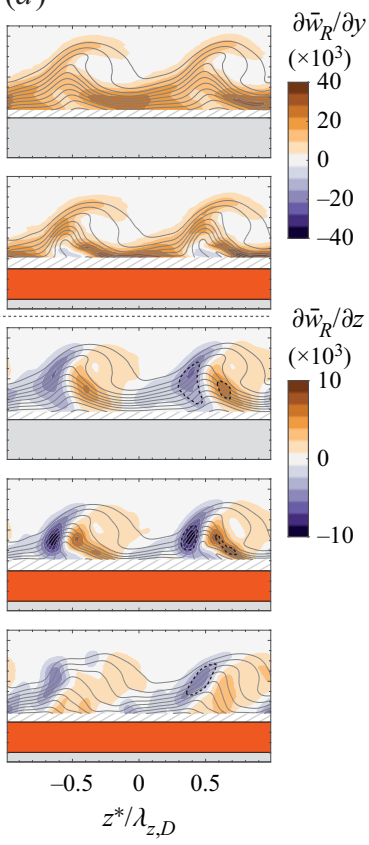

Figure 15. Contours of time-averaged velocity gradients and spanwise velocity (grey line 10 levels from 0 to 1$) z^{*}$ positive direction outboard, spatially filtered between $\sum_{n}^{5} m(0, n):(a)$ clean; $(b) A_{3} ;(c) C_{3}$; (d) $D_{3}$. (Here $\delta_{w, r}^{*}=514 \mu \mathrm{m}$ and $\lambda_{z, D}=7.5 \mu \mathrm{m}$ ). Areas $A$ and $B$ are defined in figure 16 .

appearance of a secondary set of finger vortices which develop along the shear layer on the outer side of the upwelling region (Wassermann \& Kloker 2002; Serpieri \& Kotsonis 2016).

Due to the inherent relation between unsteady secondary instabilities and mean velocity gradients, the latter are first inspected. The interaction of the stationary CFI with the FFS results in a topological modification of the structure of the CF vortices which further manifests as alterations in the vertical and spanwise velocity gradients presented in figure 15 . As already shown, downstream of the FFS edge $\left(x / c_{x}=0.204\right.$, figure $\left.9 \mathrm{II} b-\mathrm{II} c\right)$ the sudden and abrupt increase in $\bar{w}_{z}$ leads to a substantial decrease in the boundary layer momentum thickness $\left(\delta_{w}^{*}\right.$, figure $\left.6 c\right)$ when compared with the clean configuration. This transfer of high-momentum fluid towards the wall increases the vertical gradient $\left(\partial \bar{w}_{R} / \partial y\right)$ near the wall in the downwelling region of the CF vortices as shown in figure 15 (II $b-\mathrm{II} d$ ). In addition, a localised region of negative vertical gradient $\left(\partial \bar{w}_{R} / \partial y\right)$ is found at the centre of the upwelling region near the wall for the moderate $\left(C_{3}\right)$ and high $\left(D_{3}\right)$ steps. While the inclusion of the steps produces notable effects on the vertical gradient near the wall, the effect is largely minimal at the cusp of the stationary CFI (area $C$ ), where type-II secondary instability is expected to grow. This behaviour reconciles with the observations in $\S 4.2$ where the major deflection and shearing motion experienced by the stationary CF vortices was identified to be largely oriented in the spanwise direction. 


\section{A.F. Rius-Vidales and M. Kotsonis}

The strong changes in amplitude as well as spanwise shearing motion of the stationary $\mathrm{CF}$ vortices past the step edge, results in noticeable changes in the spanwise velocity gradients, which are predominantly located at the outer and inner sections of the upwelling regions. In contrast to the vertical gradient, changes in the spanwise gradient are global and affect both positive (i.e. inner, area $A$ in figure $15 \mathrm{III} a$ ) and negative (i.e. outer, area $B$ in figure $15 \mathrm{III} a$ ) gradients. Particularly the outer spanwise gradients have been typically associated with the growth of type-I secondary instability. In all monitored FFS cases, the step induces higher gradient levels than in the clean configuration near the step (figure 15IV $a-\mathrm{IV} d$ ). This influence is already evident slightly upstream of the step as shown in figure 15 (III $a-\mathrm{III} d)$.

While the intensification of the spanwise gradient is consistently observed for all cases at the vicinity of the step, the downstream development of $\partial \bar{w}_{R} / \partial z$ is highly dependent on the step height. In the case of the highest FFS $\left(D_{3}\right)$, the interaction of the stationary CFI with the step leads to an abrupt amplification, saturation and breakdown of the CF vortex structure as shown in figure $15(\mathrm{Vd})$. The interaction of the stationary CFI with the moderate step case $\left(C_{3}\right)$ follows a similar trend as the $\mathrm{CF}$ vortices experience a strong amplification near the step followed by saturation. This leads to a loss in spanwise coherence and decrease in the intensity of the spanwise gradients (figure $15 \mathrm{IV} c-\mathrm{VI} c$ ) prior to laminar breakdown, which occurs at $x / c_{x} \approx 0.27$ (figure $3 a$ ).

In contrast, for the small FFS cases $A_{3}$ and $B_{3}$ (not shown in figure 15) the CF vortices do not experience breakdown in this streamwise region. Yet, the spanwise gradient experiences a significant decay farther downstream of the step. Particularly for the shallowest case $A_{3}$, the spanwise gradient at $x / c_{x}=0.239$ (figure $15 \mathrm{VI} b$ ) is in fact rendered lower than the corresponding clean case at the same streamwise location. This is a direct consequence of the stabilisation and decrease in amplitude of the stationary CFI, as identified in figure 14 .

The significant changes on both vertical and spanwise velocity gradients due to the step can further be associated with the development and growth of unsteady shear layer instabilities, widely acknowledged to play an important role in the breakdown of CFI-dominated flows. Figure 16 presents a set of contour plots of spanwise temporal velocity fluctuations $\left(\sigma w_{R}\right)$ at selected streamwise locations upstream and downstream of the FFS location. Time-resolved hot-wire anemometry (known as HWA) measurements, on the same configuration as the present clean case and similar flow conditions by Serpieri \& Kotsonis $(2016,2018)$, identified type-I/type-II instabilities in the frequency range between 3.5 and $8 \mathrm{kHz}$ and type-III modes in the range between 350 and $550 \mathrm{~Hz}$, albeit for a lower DRE amplitude. Considering the low repetition-rate of PIV acquisitions employed in the present study (i.e. $15 \mathrm{~Hz}$ ), spectral analysis and frequency filtering are not applicable. Nevertheless, the long sampling time (i.e. $80 \mathrm{~s}$ ) ensures that the fluctuating velocity field represents an ensemble of both low- and high-frequency disturbances, which can be considered temporally uncorrelated. Furthermore, these instabilities are strongly localised within the structure of the stationary $\mathrm{CFI}$, allowing direct evaluation of the development of each type.

The results for the clean baseline case in figure 16(I $a-\mathrm{VI} a)$, show a local maximum of fluctuations at the location corresponding to a type-III mode ( $A$ in figure 16I $a$ ). Monitoring a downstream location (figure $16 \mathrm{Va}$ ) indicates a decaying interaction between weak travelling CF modes and the forced stationary CF vortices, as the magnitude of the fluctuations in this region decrease. To better illustrate this decaying behaviour, figure 17 presents average values of spanwise velocity gradient and temporal fluctuation amplitude extracted within areas $A$ and $B$ indicated in figures 15 and 16. The two extraction areas are 
$\square$ Model surface $\square$ FFS $\square$ No data

(a)

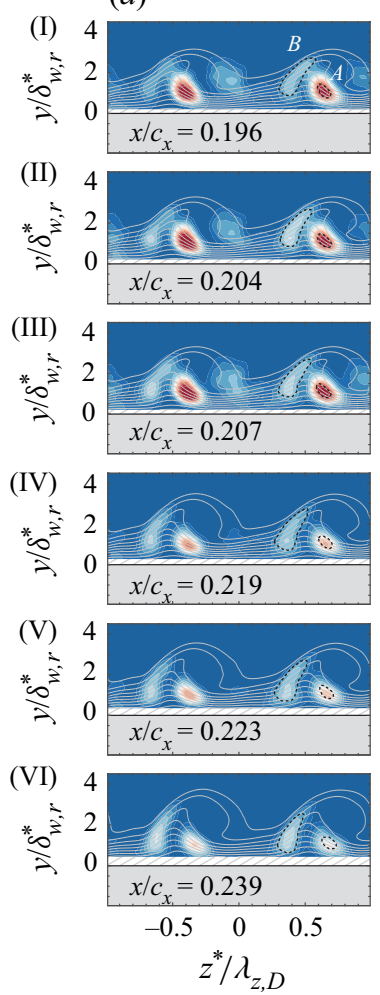

(b)
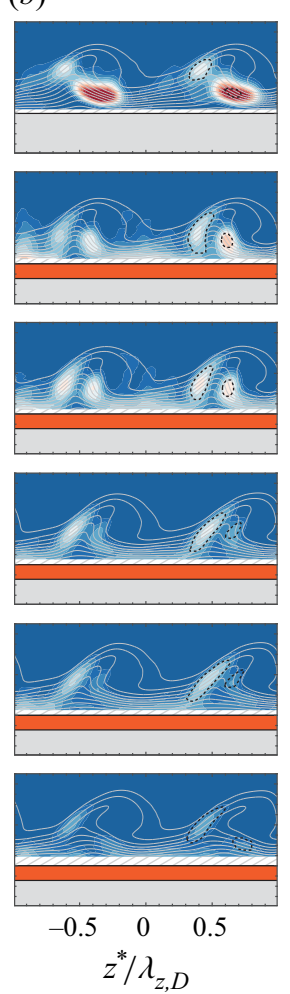

(c)
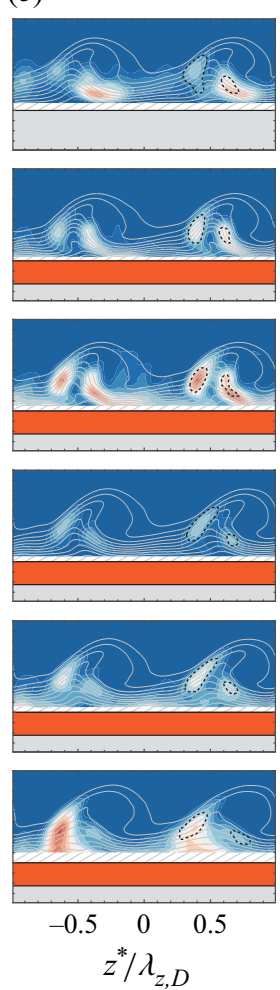

(d)
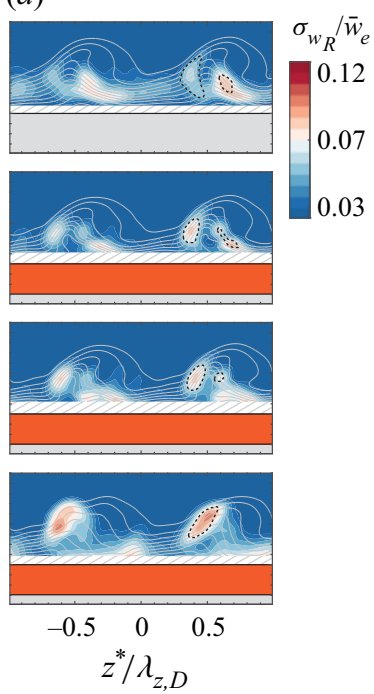

Figure 16. Contours of spanwise temporal velocity fluctuations and spanwise velocity (grey line 10 levels from 0 to 1$), z^{*}$ positive direction outboard spatially filtered between $\sum_{n}^{5} m(0, n):(a)$ clean; $(b) A_{3} ;(c) C_{3} ;(d) D_{3}$. (Here $\delta_{w, r}^{*}=514 \mu \mathrm{m}$ and $\lambda_{z, D}=7.5 \mu \mathrm{m}$ ). Extraction areas $A$ and $B$ are defined as isolines of $85 \%$ of the local maximum amplitude of unsteady fluctuations on the inner and outer side of the upwelling region.

defined for each plane and step case as isolines of $85 \%$ of the local maximum amplitude of unsteady fluctuations on the inner and outer side of the upwelling region. Naturally, values corresponding to area $A$ refer to positive spanwise gradients and type-III dominated fluctuations, while values corresponding to area $B$ refer to negative gradients and type-I dominated fluctuations.

Figure $17(b)$ confirms the decay in the temporal velocity fluctuations $\left(\sigma w_{R}\right)$ in region $A$ corresponding to type-III instabilities in the clean baseline case (black line) in the range of $x / c_{x}>0.2$. The addition of small $\left(A_{3}\right)$ to moderate $\left(C_{3}\right)$ steps result in a further reduction of velocity fluctuations $\left(\sigma w_{R}\right)$ in this region. Evidently, the addition of the FFS leads to a reduction in the interaction between travelling and stationary CF modes. Furthermore, it is striking to note that this stabilising effect is already active upstream of the FFS (compare figure 16I $a-\mathrm{I} d$ ). This behaviour strongly suggests a change in stability characteristics of the incoming boundary layer. Considering the findings described in figure 14 , for the same streamwise range $\left(0.18<x / c_{x}<0.2\right)$, the primary stationary CFI appears to destabilise and increase in amplitude. The concurrent dampening of type-III modes can then be associated with a combination of (possibly conflicting) linear effects (i.e. change of the stability of the mean boundary layer due to the FFS modification) and nonlinear interaction between stationary and travelling modes. The exact identification of 


\section{A.F. Rius-Vidales and M. Kotsonis}
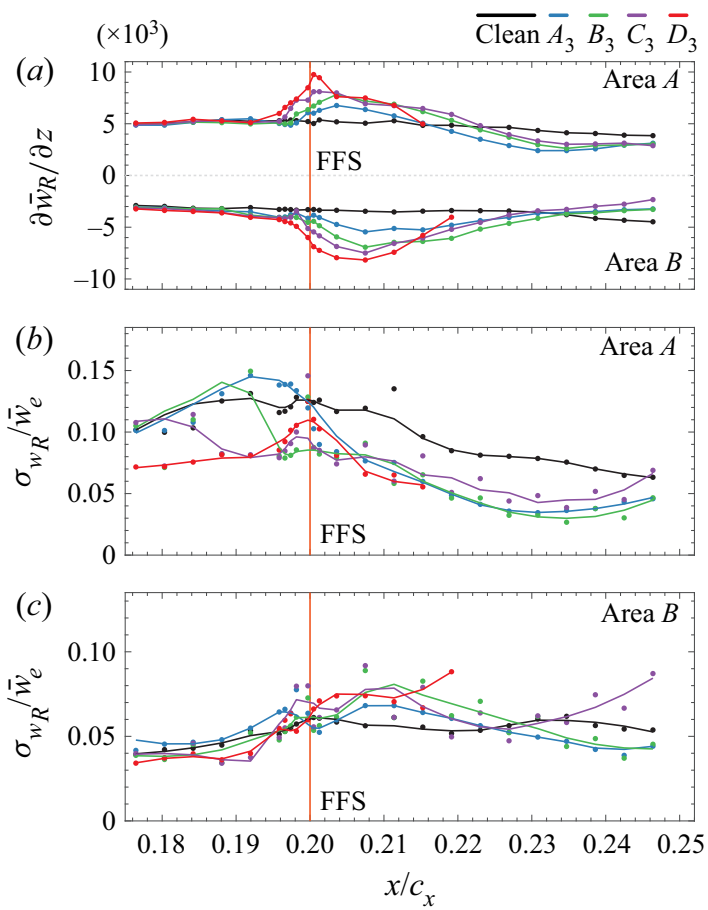

Figure 17. Streamwise evolution of averaged extracted values in areas $A$ and $B$ (defined in figures 15 and 16): (a) positive (area $A$ ) and negative (area $B$ ) averaged spanwise velocity gradients; $(b)$ averaged velocity fluctuations in area $A ;(c)$ averaged velocity fluctuations in area $B$.

the relative significance of these two effects requires a combination of parameter variations in high-resolution experiments as well as accurate numerical simulations in the near-step region.

Considering the development of secondary instabilities, detailed experimental studies (White \& Saric 2005; Serpieri \& Kotsonis 2016) have shown that as the stationary vortices saturate, the strong mean flow distortion results in the development of streamwise and spanwise velocity shears. These give rise to high-frequency inviscid instabilities of Kelvin-Helmholtz type. In the present study, the primary CFI arrives at the FFS location at a relatively high and constant amplitude of approximately $13 \%$ of the local free stream (figure 14), signalling saturation levels. Consequently, a noticeable increase in the velocity fluctuations in the region corresponding to the type-I mode (area $B$ in figure 16I $a$ ) is registered. The clean baseline case in figure 17(c) shows an increase in the velocity fluctuations $\left(\sigma w_{R}\right)$ in this region. Moreover, the addition of an FFS appears to affect the development of these fluctuations considerably. The results in figure 17(c) show an increase in velocity fluctuations $\left(\sigma w_{R}\right)$ with respect to the clean configuration in the region associated with the type-I secondary instability. This is consistent for all step cases in the region directly downstream of the FFS location $\left(x / c_{x} \approx 0.21\right)$. For the highest step height $\left(D_{3}\right)$ this increase correlates well with the breakdown of the CF vortices presented in figure $16(\mathrm{IV} d)$. For the moderate step height $\left(C_{3}\right)$, the velocity fluctuations $\left(\sigma w_{R}\right)$ in the region associated with the type-I mode increase even further for $x / c_{x}>0.22$ with respect to the clean configuration as shown in figure $16(\mathrm{~V} c$ and $\mathrm{VI} c)$. This amplification of the secondary instability correlates well with the anticipation of the laminar-turbulent transition, presented in figure 3 for the respective cases. 


\section{FFS impact on the development of crossflow instability}

In contrast, for the smaller FFS cases $\left(A_{3}\right.$ and $\left.B_{3}\right)$ the velocity fluctuations $\left(\sigma w_{R}\right)$ associated with the type-I mode decrease downstream of the FFS location $\left(x / c_{x}>0.22\right)$ to a level lower than the corresponding level in the clean baseline configuration, compare figure 16(VI $a-\mathrm{VI} b)$. Moreover, the reduction in $\sigma w_{R}$ presented in figure 17(c) correlates well with a decrease in the spanwise gradients in region B as shown in figure $17(a)$. High-resolution and time-resolved measurements are required to identify the spectral content and further confirm the origin of the observed velocity fluctuations. In addition, such future dedicated studies will indicate the connection, if any, to the vortex-shedding mechanisms proposed by Eppink (2020b). The unsteady behaviour reconciles sufficiently with the overall delay of transition presented in $\S 3.1$ for the small FFS $\left(A_{3}\right)$ and provides a first-order insight into the possible transition delay mechanisms pivoting on the reduction of the spanwise gradients and the stabilisation of the type-I secondary instability.

\section{Concluding remarks}

The attainment of extended regions of laminar flow in the boundary layer of high subsonic commercial transport aircraft is highly susceptible to the mechanical smoothness of aerodynamic surfaces. Previous research in CFI-dominated flows revealed a significant influence on the laminar-turbulent transition behaviour by two-dimensional surface irregularities in the form of steps and gaps.

This work focuses exclusively on FFSs. Most published studies on this type of surface irregularity have focused on formulating appropriate criteria to determine the critical step height, which do not result in premature transition. Nevertheless, a few recent studies have highlighted unresolved physical aspects governing the FFS-CFI interaction which necessitate detailed flow diagnostics before a universal model or criterion can be used for the design of practical laminar flow components and application of LFC techniques.

In particular, Eppink (2020b) and Rius-Vidales \& Kotsonis (2020) associated the impact of the FFS on the development and transition of the CFI with the amplitude of the incoming $\mathrm{CF}$ vortices. Consequently, the present work extends the current investigation of the FFS-CFI interaction to cases where the CF vortices arrive with high amplification to the step location.

The main findings of this work indicate strong topological changes on swept wing flows due to FFS, manifesting in a pronounced outboard-inboard-outboard motion which can be potentially linked to the streamwise development of the CFI instability. When comparing with the clean baseline configuration, as the boundary layer flow approaches the step there is a clear deceleration in the boundary layer flow (i.e. adverse pressure gradient) and the stationary $\mathrm{CF}$ vortices experience an outboard spanwise motion. Reaching the FFS, the boundary layer flow is redirected by the FFS resulting in a strong vertical velocity ejection. This behaviour correlates well with the measured amplification of the stationary CF vortices upstream of the FFS location.

Downstream of the FFS edge, the stationary CF vortices experience an abrupt inboard spanwise motion, linked to the localised favourable pressure gradient, reaching their maximum amplification and strong mean flow distortion. Farther downstream, as the flow recovers to nominal pressure conditions, the $\mathrm{CF}$ vortices experience a gradual outboard spanwise motion (i.e. adverse pressure gradient) and a substantial amplitude reduction, even below the level pertaining to the clean configuration. For the smaller step cases, a second region of growth of the $\mathrm{CF}$ vortices is observed, enhanced by the nominal favourable pressure distribution of the wing.

In agreement with previous studies (Eppink 2020b; Rius-Vidales \& Kotsonis 2020), an amplification of higher spanwise harmonics of the stationary CF mode at the step 


\section{A.F. Rius-Vidales and M. Kotsonis}

location has been observed. The observed behaviour suggests the dominance of indirect harmonic growth due to nonlinear forcing of the primary mode at the step location. For the smaller FFS cases studied in this work, the step has a local stabilising effect on the primary stationary mode and its harmonics. For the higher step case transition occurs shortly downstream of the step location.

The analysis of the unsteady disturbances revealed a reduction in spanwise velocity temporal fluctuations $\left(\sigma w_{R}\right)$ due to the step, in the region associated with type-III travelling instabilities. The amplification experienced by the primary CFI mode due to the step is sufficient to minimise the nonlinear interaction between the stationary and travelling $\mathrm{CF}$ vortices. On the other hand, for all the FFS cases, the spanwise velocity temporal fluctuations $\left(\sigma w_{R}\right)$ in the region associated with type-I secondary instability, show a substantial increase past the step edge as the primary stationary CFI mode and its higher harmonics reach their maximum amplification. This is strongly correlated with the modifications imparted on the spanwise gradients of time-averaged velocity, widely acknowledged as the driver for type-I instabilities. Downstream of this location, the behaviour strongly differs, depending on the considered step height. For the largest step height, an immediate breakdown of the $\mathrm{CF}$ vortices and onset of turbulent flow occurs, likely related to the explosive growth of type-I fluctuations. For the moderate FFS case, the $\sigma w_{R}$ fluctuations slightly decrease before rapidly amplifying, reaching higher levels than the clean baseline configuration by the end of the measurement domain.

In contrast, for the smallest FFS case, a substantial decrease in the spanwise velocity gradient and $\sigma w_{R}$ fluctuations in the region associated with type-I secondary instability is achieved, reaching levels lower than the clean baseline configuration by the end of the PIV measurement domain. This behaviour reflects an unprecedented transition delay effect due to a small FFS, holding potential for understanding and facilitating the future design of laminar flow components.

Acknowledgements. The authors express their gratitude to S. Bernardy, E. Langedijk, P. Duyndham and N. Van Beek for their technical support and to the TU Delft Aerospace Materials and Structures Laboratory for providing advice and equipment to manufacture and measure the surface irregularity add-ons. In addition, the contribution of our colleagues G. Zoppini, K. Peng and J. Casacuberta during the execution of the experiments and the discussion of the results is greatly appreciated. Finally, the authors would like to acknowledge the anonymous referees for their valuable comments.

Declaration of interests. The authors report no conflict of interest.

Author ORCIDs.

(1) Alberto F. Rius-Vidales https://orcid.org/0000-0003-4342-0278;

D. Kotsonis https://orcid.org/0000-0003-0263-3648.

\section{REFERENCES}

ARNAL, D. \& ARChAmbaud, J.P. 2008 Laminar-turbulent transition control: Nlf, lfc, hlfc. In Advances in Laminar-Turbulent Transition Modeling. AVT-151 RTO AVT/VKI, Lecture Series. VKI Inst, Belgium.

ARnal, D. \& CASAlis, G. 2000 Laminar-turbulent transition prediction in three-dimensional flows. Prog. Aerosp. Sci. 36 (2), 173-191.

Beguet, S., Perraud, J., Forte, M. \& Brazier, J.-P.. 2017 Modeling of transverse gaps effects on boundary-layer transition. AIAA J. Aircraft 54 (2), 794-801.

BIPPES, H. 1999 Basic experiments on transition in three-dimensional boundary layers dominated by crossflow instability. Prog. Aerosp. Sci. 35 (4), 363-412.

Bonfigli, G. \& KLOKER, M. 2007 Secondary instability of crossflow vortices: validation of the stability theory by direct numerical simulation. J. Fluid Mech. 583, 229-272.

BRANDT, L. 2014 The lift-up effect: the linear mechanism behind transition and turbulence in shear flows. Eur. J. Mech. B/Fluids 47, 80-96. 


\section{FFS impact on the development of crossflow instability}

Cooke, E.E., Mughal, M.S., Sherwin, S., Ashworth, R. \& Rolston, S. 2019 Destabilisation of stationary and travelling crossflow disturbances due to steps over a swept wing. AIAA Aviation 2019 Forum. AIAA Paper 2019-3533.

Costantini, M., Risius, S. \& Klein, C. 2015 Experimental investigation of the effect of forward-facing steps on boundary layer transition. Procedia IUTAM 14, 152-162.

Costantini, M., Risius, S. \& KLEIN, C. 2018 Non-adiabatic surface effects on step-induced boundary-layer transition. Flow Turbul. Combust. 100 (4), 1145-1177.

Crawford, B.K., Duncan, G.T., Tufts, M.W., Saric, W.S. \& ReEd, H.L. $2015 a$ Effects of step-excrescence location on swept-wing transition. 53rd AIAA Aerospace Sciences Meeting. AIAA Paper 2015-1233.

Crawford, B.K., Duncan, G.T., West, D.E. \& SARIC, W.S. $2015 b$ Robust, automated processing of IR thermography for quantitative boundary-layer transition measurements. Exp. Fluids 56 (7), 149.

Crouch, J.D., Kosorygin, V.S. \& NG, L.L. 2006 Modeling the effects of steps on boundary-layer transition. In IUTAM Symposium on Laminar-Turbulent Transition. Fluid Mechanics and its Applications (ed. R. Govindarajan), vol. 78, pp. 37-44. Springer.

CROUCH, J.D. \& Kosorygin, V.S. 2020 Surface step effects on boundary-layer transition dominated by Tollmien-Schlichting instability. AIAA J. 58 (7), 2943-2950.

Crouch, J.D.., Kosorygin, V.S.. \& Sutanto, M.I. 2020 Modeling gap effects on transition dominated by Tollmien-Schlichting instability. AIAA Aviation 2020 Forum. AIAA Paper 2020-3075.

Dagenhart, J., Stack, J., Saric, W. \& Mousseux, M. 1989 Crossflow-vortex instability and transition on a $45^{\circ}$ swept wing. 20th Fluid Dynamics, Plasma Dynamics and Lasers Conference. AIAA Paper 1989-1892.

Downs, R.S. \& White, E.B. 2013 Free-stream turbulence and the development of crossflow disturbances. J. Fluid Mech. 735, 347-380.

Drake, A., Bender, A., Korntheuer, A., Westphal, R., Rohe, W., Dale, G., McKeon, B. \& GERASCHCHENKo, S. 2010 Step excrescence effects for manufacturing tolerances on laminar flow wings. 48th AIAA Aerospace Sciences Meeting Including the New Horizons Forum and Aerospace Exposition. AIAA Paper 2010-375.

Drake, A., Bender, A.M. \& Westphal, R.V. 2008 Transition due to surface steps in the presence of favorable pressure gradients. 26th AIAA Applied Aerodynamics Conference. AIAA Paper 20087334.

Drake, A., Westphal, R.V., Zuniga, F.A., Kennelly, R.A. JR. \& KogA, D.J. 1996 Wing leading edge joint laminar flow test. Technical memorandum 4762. NASA.

Duncan, G.T., Crawford, B.K., Tufts, M.W., Saric, W.S. \& Reed, H.L. $2014 a$ Effects of step excrescences on a swept wing in a low-disturbance wind tunnel. 52nd Aerospace Sciences Meeting. AIAA Paper 2014-0910.

Duncan, G.T., Crawford, B.K., Tufts, M.W., Saric, W.S. \& ReEd, H.L. $2014 b$ Flight experiments on the effects of step excrescences on swept-wing transition. Intl J. Engng Syst. Model. Simul. 6 (3-4), 171-180.

EDELMANN, C.A. \& Rist, U. 2015 Impact of forward-facing steps on laminar-turbulent transition in transonic flows. AIAA J. 53 (9), 2504-2511.

EPPINK, J.L. 2017 Stereo particle image velocimetry measurements of transition downstream of a foward-facing step in a swept-wing boundary layer. 55th AIAA Aerospace Sciences Meeting. AIAA Paper 2017-0306.

EPPINK, J.L. 2018 The effect of forward-facing steps on stationary crossflow instability growth and breakdown. 2018 AIAA Aerospace Sciences Meeting. AIAA Paper 2018-0817.

EPPINK, J.L. $2020 a$ High-frequency secondary instabilities downstream of a forward-facing step. AIAA Scitech 2020 Forum. AIAA Paper 2020-2243.

EPPINK, J.L. $2020 b$ Mechanisms of stationary crossflow instability growth and breakdown induced by forward-facing steps. J. Fluid Mech. 897, A15.

EPPINK, J.L. \& CASPER, C. 2019 Effects of forward-facing step shape on stationary crossflow instability growth and breakdown. AIAA Aviation 2019 Forum. AIAA Paper 2019-3532.

EPPINK, J.L., WLEZIEN, R.W., King, R.A. \& CHOUdHARI, M. 2018 Interaction of a backward-facing step and crossflow instabilities in boundary-layer transition. AIAA J. 56 (2), 497-509.

GERHARDT, H.A. 1967 Section 10: waviness and surface smoothness criteria. In Final Report on LFC Aircraft Design Data Laminar Flow Control Demonstration Program. Norair report no. NOR-67-136.

HAYNES, T.S. \& REED, H.L. 2000 Simulation of swept-wing vortices using nonlinear parabolized stability equations. J. Fluid Mech. 405, 325-349. 


\section{A.F. Rius-Vidales and M. Kotsonis}

Holmes, B.J., Obara, C.J., Martin, G.L. \& Domack, C.S. 1985 Manufacturing tolerances for natural laminar flow airframe surfaces. SAE Tech. Paper. 850863.

Ivanov, A.V. \& Mischenko, D.A. 2019 Delay of laminar-turbulent transition on swept-wing with help of sweeping surface relief. AIP Conf. Proc. 2125 (1), 030041.

Joslin, R.D. 1998 Aircraft laminar flow control. Annu. Rev. Fluid Mech. 30 (1), 1-29.

LANDAHL, M.T. 1980 A note on an algebraic instability of inviscid parallel shear flows. J. Fluid Mech. 98 (2), 243-251.

Malik, M.R., Li, F., Choudhari, M.M. \& ChanG, C.-L. 1999 Secondary instability of crossflow vortices and swept-wing boundary-layer transition. J. Fluid Mech. 399, 85-115.

Marxen, O., LAng, M., Rist, U., Levin, O. \& Henningson, D.S. 2009 Mechanisms for spatial steady three-dimensional disturbance growth in a non-parallel and separating boundary layer. J. Fluid Mech. 634, 165-189.

Messing, R. \& KLOKER, M.J. 2010 Investigation of suction for laminar flow control of three-dimensional boundary layers. J. Fluid Mech. 658, 117-147.

Perraud, J., Arnal, D. \& Kuehn, W. 2014 Laminar-turbulent transition prediction in the presence of surface imperfections. Intl J. Engng Syst. Model. Simul. 6 (3-4), 162-170.

Perraud, J. \& SEraudie, A. 2000 Effects of steps and gaps on 2D and 3D transition. European Congress on Computational Methods in Applied Science and Engineering, ECCOMAS Paper 634.

RAFFEL, M. \& MERZ, C.B. 2014 Differential infrared thermography for unsteady boundary-layer transition measurements. AIAA J. 52 (9), 2090-2093.

Raffel, M., Merz, C.B, Schwermer, T. \& Richter, K. 2015 Differential infrared thermography for boundary layer transition detection on pitching rotor blade models. Exp. Fluids 56, 30.

Reibert, M., SARIC, W., CARRILlo, R. JR. \& CHAPMAN, K. 1996 Experiments in nonlinear saturation of stationary crossflow vortices in a swept-wing boundary layer. In 34th AIAA Aerospace Sciences Meeting and Exhibit, AIAA Paper 1996-184.

RiUs-VidAlES, A.F. \& Kotsonis, M. 2020 Influence of a forward facing step surface irregularity on swept wing transition. AIAA J. 58 (12), 5243-5253.

Rius-Vidales, A.F., Kotsonis, M., Antunes, A.P. \& Cosin, R. 2018 Effect of two-dimensional surface irregularities on swept wing transition: forward facing steps. 2018 Fluid Dynamics Conference. AIAA Paper 2018-3075.

RizZetTA, D.P. \& Visbal, M.R. 2014 Numerical simulation of excrescence generated transition. AIAA J. 52 (2), 385-397.

RizzeTtA, D.P. \& Visbal, M.R. 2017 Plasma-based control of excrescence-generated transition on a swept wing. AIAA J. 55 (5), 1610-1621.

Saeed, T.I., Mughal, M.S. \& Morrison, J. 2016 The interaction of a swept-wing boundary layer with surface excrescences. 54th AIAA Aerospace Sciences Meeting. AIAA Paper 2016-2065.

SARIC, W., CARrillo, R. JR. \& ReiberT, M. 1998 Leading-edge roughness as a transition control mechanism. 36th AIAA Aerospace Sciences Meeting and Exhibit, AIAA Paper 1998-781.

SARIC, W.S., CARPenter, A.L. \& REED, H.L. 2011 Passive control of transition in three-dimensional boundary layers, with emphasis on discrete roughness elements. Phil. Trans. R. Soc. Lond. A 369 (1940), 1352-1364.

SARIC, W.S., REED, H.L. \& White, E.B. 2003 Stability and transition of three-dimensional boundary layers. Annu. Rev. Fluid Mech. 35 (1), 413-440.

SARIC, W.S., West, D.E., Tufts, M.W. \& REED, H.L. 2019 Experiments on discrete roughness element technology for swept-wing laminar flow control. AIAA J. 57 (2), 641-654.

Schrauf, G. 2005 Status and perspectives of laminar flow. Aeronaut. J. (1102) 109, 639-644.

SERPIERI, J. 2018 Cross-flow instability flow diagnostics and control of swept wing boundary layers. PhD thesis, Delft University of Technology, pp. 28-29.

SERPIERI, J. \& Kotsonis, M. 2015 Design of a swept wing wind tunnel model for study of crossflow instability. 33rd AIAA Applied Aerodynamics Conference. AIAA Paper 2015-2576.

SERPIERI, J. \& Kotsonis, M. 2016 Three-dimensional organisation of primary and secondary crossflow instability. J. Fluid Mech. 799, 200-245.

SERPIERI, J. \& Kotsonis, M. 2018 Conditioning of unsteady crossflow instability modes using dielectric barrier discharge plasma actuators. Exp. Therm. Fluid Sci. 93, 305-318.

Serpieri, J., YADAla Venkata, S. \& Kotsonis, M. 2017 Conditioning of crossflow instability modes using dielectric barrier discharge plasma actuators. J. Fluid Mech. 833, 164-205.

Tufts, M.W., Reed, H.L., Crawford, B.K., Duncan, G.T. \& SARIC, W.S. 2017 Computational investigation of step excrescence sensitivity in a swept-wing boundary layer. AIAA J. Aircraft 54 (2), 602-626. 


\section{FFS impact on the development of crossflow instability}

Ustinov, M. \& IVAnov, A. 2018 Cross-flow dominated transition control by surface micro-relief. AIP Conf. Proc. 2027 (1), 020013.

WANG, Y.X. \& GASTER, M. 2005 Effect of surface steps on boundary layer transition. Exp. Fluids 39, 679-686.

Wassermann, P. \& Kloker, M. 2002 Mechanisms and passive control of crossflow-vortex-induced transition in a three-dimensional boundary layer. J. Fluid Mech. 456, 49-84.

Wassermann, P. \& KloKer, M. 2003 Transition mechanisms induced by travelling crossflow vortices in a three-dimensional boundary layer. J. Fluid Mech. 483, 67-89.

White, E.B. \& SARIC, W.S. 2005 Secondary instability of crossflow vortices. J. Fluid Mech. 525, 275-308.

Yadala, S., Hehner, M.T., Serpieri, J., Benard, N., Dörr, P.C., Kloker, M.J. \& Kotsonis, M. 2018 Experimental control of swept-wing transition through base-flow modification by plasma actuators. J. Fluid Mech. 844, R2.

ZAHN, J. \& RIST, U. 2016 Impact of deep gaps on laminar-turbulent transition in compressible boundary-layer flow. AIAA J. 54 (1), 66-76.

ZUCCHER, S. \& SARIC, W.S. 2008 Infrared thermography investigations in transitional supersonic boundary layers. Exp. Fluids 44, 145-157.

Zuniga, F.A., Drake, A, Kenelly, R.A. JR., Koga, D.J. \& Westphal, R.V. 1994 Transonic flight test of a laminar flow leading edge with surface excrescences. Biennial Flight Test Conference. AIAA Paper 1994-2142. 